\title{
Assessment of an Increase in Boron and Arsenic Concentrations at the Discharge Area of Na-Borate Mine (Kirka-Eskisehir, Turkey)
}

\author{
Galip Yuce* and Didem Ugurluoglu Yasin \\ Faculty of Engineering-Architecture, Department of Geological Engineering, Eskisehir Osmangazi University, Eskisehir, Turkey
}

Received 23 February 2012, accepted 10 May 2012

\begin{abstract}
The Province of Kirka (Eskisehir, Turkey), located in the catchment of the Seydisuyu Plain, has one of the largest Naborate deposits in the world. However, boron concentrations in surface and ground waters downstream of Kirka Province, the Seyitgazi Plain, have increased over the past twenty years. Seyitgazi plain has been widely irrigated from groundwater and Seydisuyu River. In this respect, quality of the river water directly affects groundwater quality in the Seyitgazi Plain since river water is the main supplier for irrigation water in the Seyitgazi plain in addition to groundwater which is extracted from the wells drilled in the Seyitgazi Plain. Thus, any mining activity built in the catchment area of groundwater poses potential risk for the quality of groundwater in the discharge area.

The Seydisuyu River is fed from Catören and Kunduzlar dam reservoirs which are located in the upper reaches of the Seyitgazi Plain. Chemical analysis of surface waters $(n=102)$ and groundwaters $(n=136)$ showed that boron concentrations in groundwater of the Seyitgazi Plain increased up to three to four times due to infiltration into the shallow aquifer system because the Seydisuyu River water is used for irrigation. Statistically, significant positive correlations were found between boron and arsenic due to deposition in the same playa lake. Since the boron bearing layers can contain arsenic minerals, arsenic can act in a tracer role for surplus boron concentrations in groundwater. Besides natural contribution, mining activities caused a substantial increase in boron and arsenic concentrations in the groundwater of the Seyitgazi Plain.
\end{abstract}

Key words: Kirka, Borax, Boron, Arsenic, Contamination, Groundwater

Citation: Yuce, G. and D. Ugurluoglu Yasin, 2012: Assessment of an increase in boron and arsenic concentrations at the discharge area of Na-borate mine (Kirka-Eskisehir, Turkey). Terr. Atmos. Ocean. Sci., 23, 703-723, doi: 10.3319/TAO.2012.05.10.01(Hy)

\section{INTRODUCTION}

Boron is an element broadly distributed with low concentration in the environment. Minable deposits of borate minerals are rare and usually found in arid regions with a geological history of volcanic and/or hydrothermal activity (Mellor 1980). Boron can leach into groundwater from rocks and soils. Depending on forming condition, arsenic may accompany boron. Boron concentration in groundwater ranges from 0.3 to $100 \mathrm{mg} \mathrm{l}^{-1}$ throughout the world (Hem 1975), while it ranges from 0.1 to $1.5 \mathrm{mg} \mathrm{l}^{1^{-1}}$ in Europe (ECETOC 1997; WHO 1998). WHO (World Health Organization) and EPA (United States Environmental Protection Agency) recommend the limit for arsenic as $10 \mu \mathrm{g} \mathrm{l}^{-1}$ for safe drinking water.

\footnotetext{
* Corresponding author

E-mail: galipyuce@gmail.com
}

Major borate deposits are found in Turkey, the USA, Argentina, Russia, Chile, China and Peru (Culver et al. 1994). The two largest producers are the USA and Turkey (WHO 1998). The borate deposits of Turkey are found mainly in five districts of Western Anatolia: Bigadiç, Kestelek, Sultançayır, Emet and Kirka Provinces. These areas were formed in a lacustrine environment during calc-alkaline volcanic activity periods in the Neogene (Palmer and Helvaci 1995, 1997). One of the commercial borate minerals is tincal, i.e., Na-borax, and is mainly found in Kirka Province, Turkey.

High boron in shallow groundwater generally originates either from upwelling geothermal waters or contamination, caused by mining acitivites (Smedley and Kinniburgh 2002; Younger et al. 2004). As a result of this, geothermal waters with high B concentrations dramatically affect the quality of shallow groundwater in Western Turkey 
(Gemici and Tarcan 2002; Vengosh et al. 2002; Baba et al. 2009). The pollution problem caused by boron mining activities has been studied before (DSI 1971, 1983; Urgüplü 1985; Onacak 1990; Wolkersdorfer 1996; Akbas 1998; Iller Bankası 2000; Rural Service of Eskisehir Research Institute 2001, 2002, 2003; Ugurluoglu 2004; Yasin and Yuce 2008).
However, the origin of boron excess in groundwater has not been well addressed.

The study area is the Kirka province which has the biggest Na-Borate deposit in the world (Fig. 1a). The two dams, Catören and Kunduzlar, are located in the discharge area of Na-borate deposit and boron mining activities. The Catören (a)

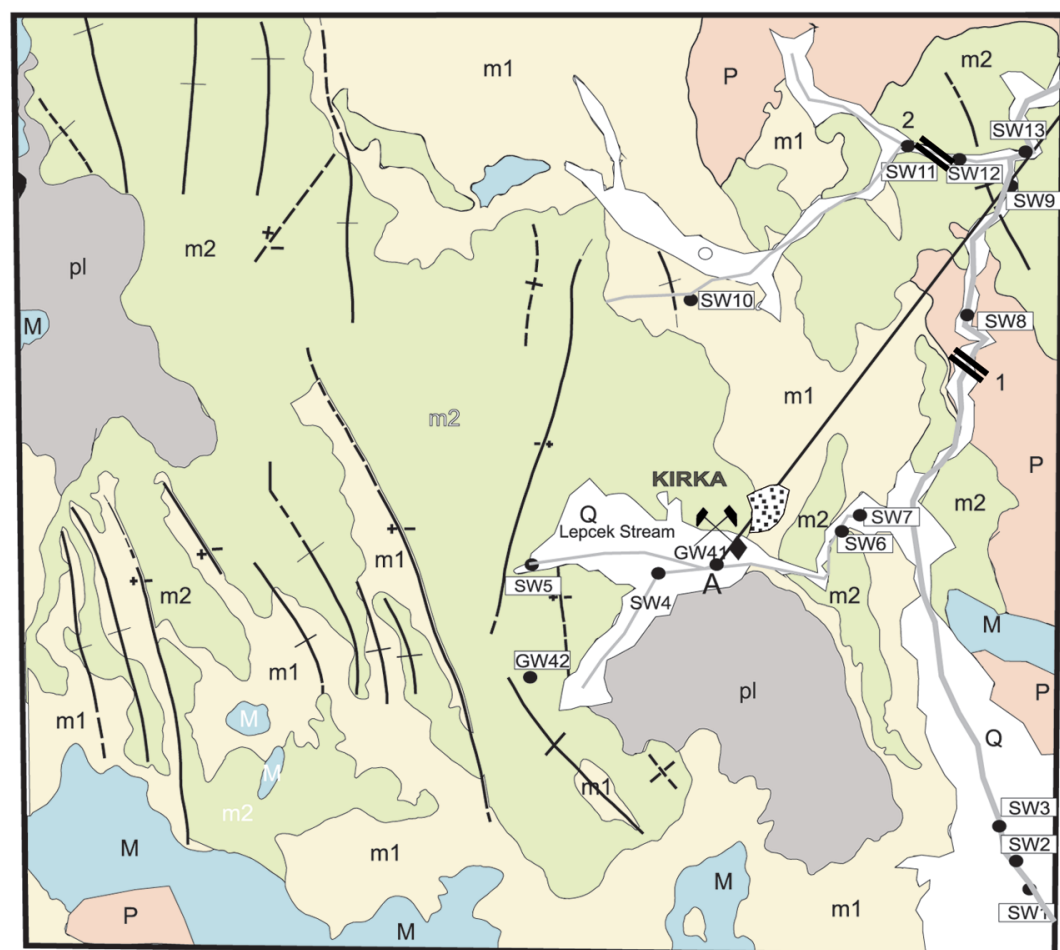

(b)

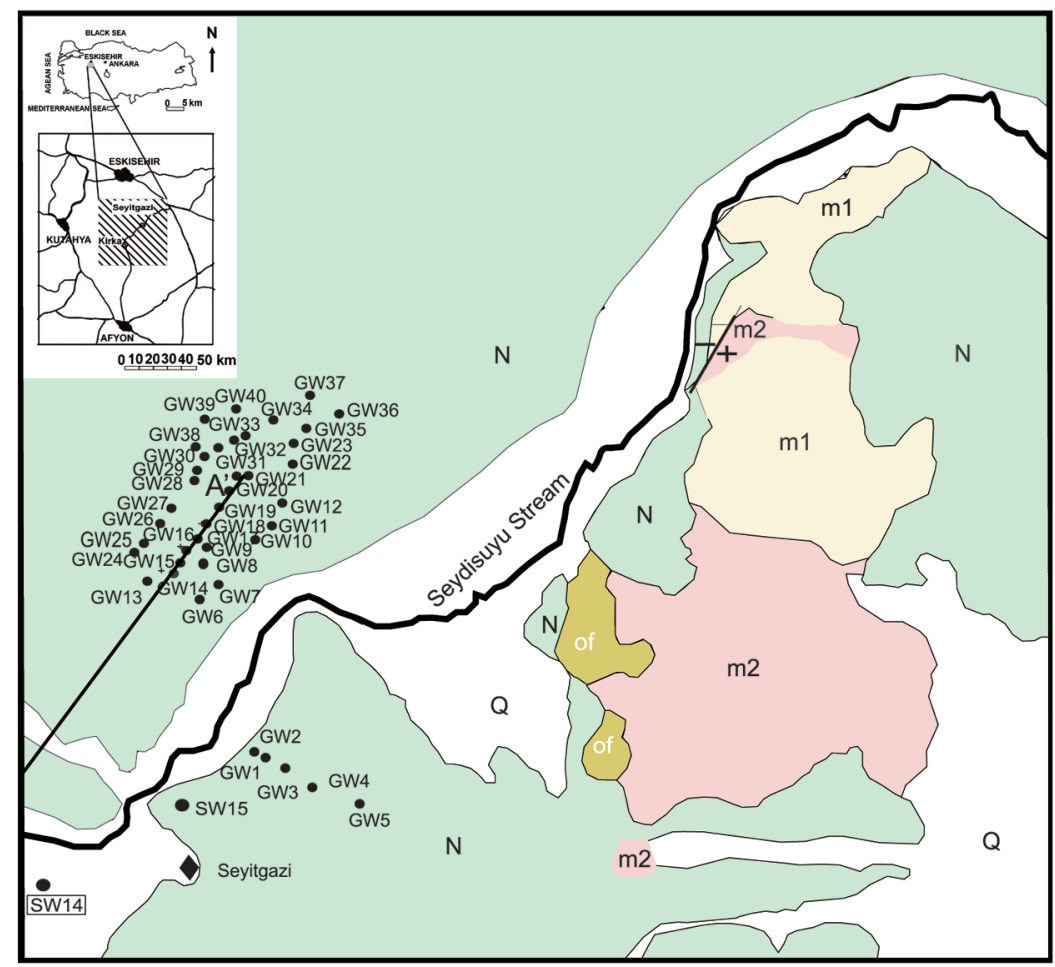

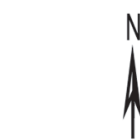
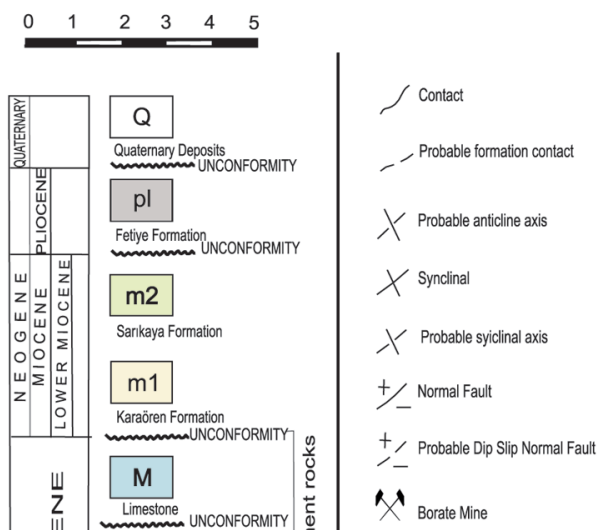

2.3. Waste Disposal Pond

1 Catören Dam

2 Kunduzlar Dam

- SAMPLING LOCATIONS

kesenler regulator

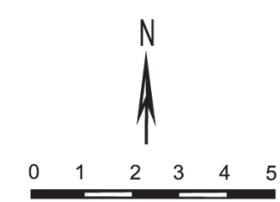

-Fault
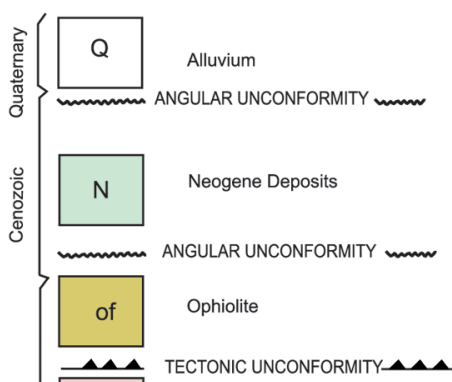

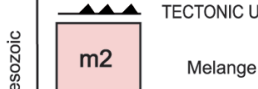

m1 Gokceyayla Formation

- SAMPLING Locations

a - a' geological cross-section

Fig. 1. (a) Geological Map of Kirka Basin (After Onacak 1990). (b) Geological Map of Seyitgazi Basin (After Ozcan et al. 1989). 
dam's water forms the Seydisuyu River which flows to the Seydisuyu Plain. The Kirka Na-borate deposit was operated as an open-pit between 1971 and 1989 without tailing ponds, since then it has still been operated as the open-pit mine with the tailing ponds. Generally, the boron concentration in surface water was generally low up to the location of the Balik Bogazi stream (SW6) except for the period of 1979 - 1980 in which the mine activities were initiated at the recharge area of the Lepcek stream. Waste rock from ore has been deposited on the surface close to the mine. However, surface water in the study area was affected by leaching/seepage from the tailing ponds at the mine site. All shallow water has been collected and stored by the abovementioned dams to supply irrigation water to the Seyitgazi Plain. After obtaining borax, resulting from the leaching process in the mining area, the solid waste has been dumped near surface water in the tailing area. There also exists two big tailings ponds to collect the leachates from the ore-deposits. Since the solid waste of Na-borate is easily soluble in water, it dissolves after rain and reaches the stream water near the Kirka mining area. Thus, boron concentration has increased in streams and creeks, especially during the wet seasons and all surface water recharge into the lake of the dams used for irrigation water in the Seyitgazi Plain. The Seyitgazi Plain is irrigated from the Catören and Kunduzlar dam lakes water as well as from wells (42 bores) that drilled to meet the deficiency of irrigation water. However, utilization of water having high boron concentration from Catören dam reservoir for irrigation purpose causes boron increment in groundwater at the discharge area, i.e., at the Seyitgazi Plain. Concordantly, boron concentration in the bottom sediments of the lake at the Catören dam was found to be 13.2 and 4 ppm for the lake at the Kunduzlar dam (Özkurt 2000). As a result, boron concentration in groundwater is higher than the allowable limit for irrigation purposes (>3 ppm).

In addition to boron, arsenic was found in higher concentrations $(>10 \mathrm{ppb})$ in groundwater samples from the bores at the discharge area of boron mine area. As is wellknown, arsenic contaminated groundwater causes a serious health problem over a long-term. In this context, Unsal and Metintas (2002) determined some health problems such as pulmonary emphysema, asthma and bronchitis in the dwellers of Kirka Province where the level of boron was found to be equal or higher than $0.6 \mathrm{mg} 1^{-1}$ were significantly greater than those in the other settlements where the level of boron was found to be lower than $0.6 \mathrm{mg} 1^{-1}$. Dissolution of the borate and a lesser amount of arsenic minerals in the boron bearing formation (Sarkkaya) or tuff through water-rock interaction, arsenic concentration increases in groundwater at the Seyitgazi Plain by use of irrigation water from the Catören dam reservoir. Groundwater extraction may also negatively affect arsenic mobilization in the downstream area of Kirka Province. Strontium was another trace element as remarkable concentrations in water resources with boron and arsenic.

To determine the hydrologic relations between water resources at the study area, in addition to hydrochemical studies, stable isotope and tritium analysis were analyzed, as well.

The aim of the study was to determine and evaluate the long-term geogenic effect of Kirka Na-borate mine activities on the quality of surface and groundwater at the discharge area, Seyitgazi Plain.

\section{GEOLOGICAL SETTINGS}

Geological maps of the study area are separately given as a recharge and discharge area in Figs. 1a and b, respectively. The basement rocks in the Kirka Plain contain Pre-Miocene units composed of metamorphics (probably Paleozoic) and Mesozoic limestone (Fig. 1a). Pre-Miocene units are overlain by Neogene units [Karaören Formation: continental and lacustrine tuff, Sarıkaya Formation: carbonate rocks, clay, borate, ignimbrite, tuff-zeolitic tuff (Helvaci and Alonso 2000), Fethiye Formation: re-sedimented tufftuffit, Quaternary deposits: alluvium] (Onacak 1990). The ignimbrite in the Kirka Region has high F and Rb concentrations; whereas the alkali lava suite has high $\mathrm{Sr}$ and $\mathrm{Ba}$ content (Floyd et al. 1998). The borate deposits formed in a lacustrine environment during periods of calc-alkaline volcanic activity in the Neogene. Actually, volcano-sedimentary sequence and alluvium form the main lithology in the Kirka area (Fig. 1a). The sequence consists predominantly of carbonate rocks, tuff, marl, tuffite, lenses of boron bands. The carbonate rocks are interbedded with various lithological units such as limestone, boron, tuff, tuffite and agglomerate as the lenses and thin bands. The basement units of the Seyitgazi Plain (Fig. 1b) are represented by Mesozoic aged Gökçeyayla formation (carbonate rocks), Melange (claystone, mudstone, sandstone), and ophiolite. The basement rocks are unconformably overlain by Neogene deposits (carbonate rocks, claystone with borate lenses, marl, agglomerate, tuff and tuffite) and Quaternary alluvium (Ozcan et al. 1989).

A stratigraphic section of the Kirka Provience and the general geologic cross-section of study area are shown in Fig. 2, respectively. The main boron mineral in the Kirka Province is borax; colemanite and ulexite are found in minor quantities. Other borate minerals such as pandermite, inyoite, meyerhofferite, tincalconite, kernite, hydroboracite, inderite, inderborite, kurnakovite, and tunnelite (Palmer and Helvaci 1995) have also been found in the Kirka Province. Sarıkaya formation is the source of borax deposit in the study area. This formation contains borate minerals that interlayered with claystones and tuffs, while a porcelanous limestone forms a cap-rock for borate zone.

The clay layers contain some volcanic tuff (frequently altered to zeolites), quartz, biotite, and feldspar. $\mathrm{Na}, \mathrm{Sr}$ and 


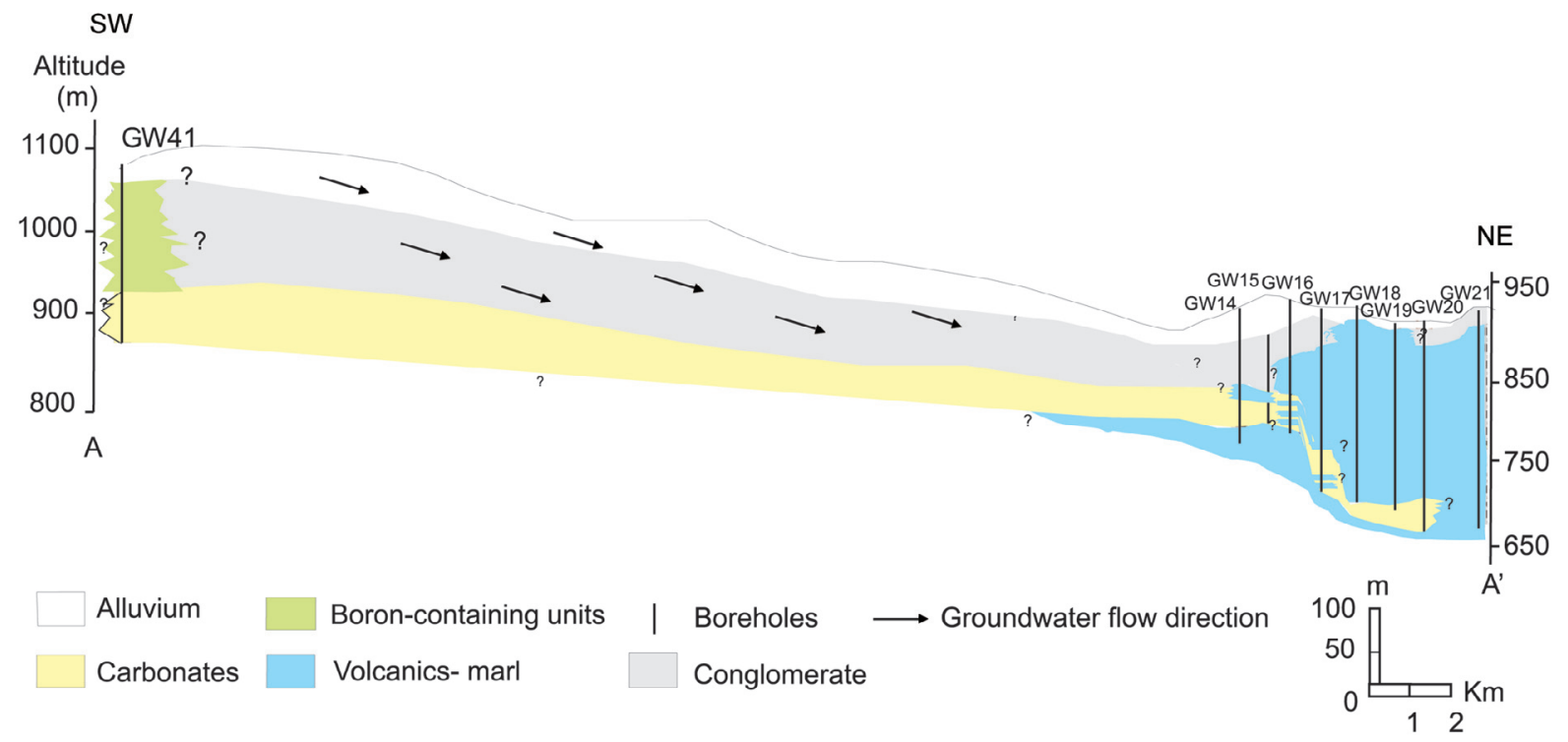

Fig. 2. Geologic cross-section of the study area. [Lithologic descriptions of the boreholes were defined according to the borehole logs provided by DSI (the Regional Directorate of State Hydraulic Works)].

$\mathrm{B}$ are originated from partial melting of lithosphere into magma and $\mathrm{Ca}$ derives from surrounding rocks, while $\mathrm{Mg}$ is sourced from ophiolite. Strontium is released to the surface because of hydrolysis of pyroclastic materials (Baysal 1972). These mixed fluids are discharged into the playa lake, concentrated by evaporation and formed borate deposits (Yalcin and Baysal 1991).

Alluvium is generally composed of fine-grained silt and sand materials along the Seydisuyu River. The sedimentary units form a poor aquifer condition because of fine detritic materials, which has lower permeability. The groundwater level depth in the study area varies from 9.5 to $32 \mathrm{~m}$ from the surface. Tuff, limestone and alluvium are aquifer units in the study area. Tuff and limestone aquifers are considered as a semi-confined aquifer while the alluvial aquifer is unconfined. The groundwater flow direction is from SW to NE. The map given in Fig. 3 shows that groundwater at the discharge area of Kirka is fed from the Seydisuyu River.

\section{METHODS}

The water samples, collected in wet and dry periods of 2007, were analyzed in the laboratories of Hacettepe University (HU), Ankara, Turkey. In addition to sampling campaigns performed in 2007, samples collected and analyzed by different governmental agencies between 1971 and 2003. In all, 102 surface waters and 136 groundwater samples were also evaluated in this paper. Groundwater samples were taken from DSI (The General Directorate of State Hydraulic Works) wells drilled in the Seyitgazi Plain. Boron contents of water samples were determined by the
Carmine method using a spectrophotometer at the governmental agency, Rural Affairs Service of Eskisehir (RASE). This method was based on the color change of the solution (carminic acid and concentrated $\mathrm{H}_{2} \mathrm{SO}_{4}$ ) depending on the boron content of water samples. Intensity of color change

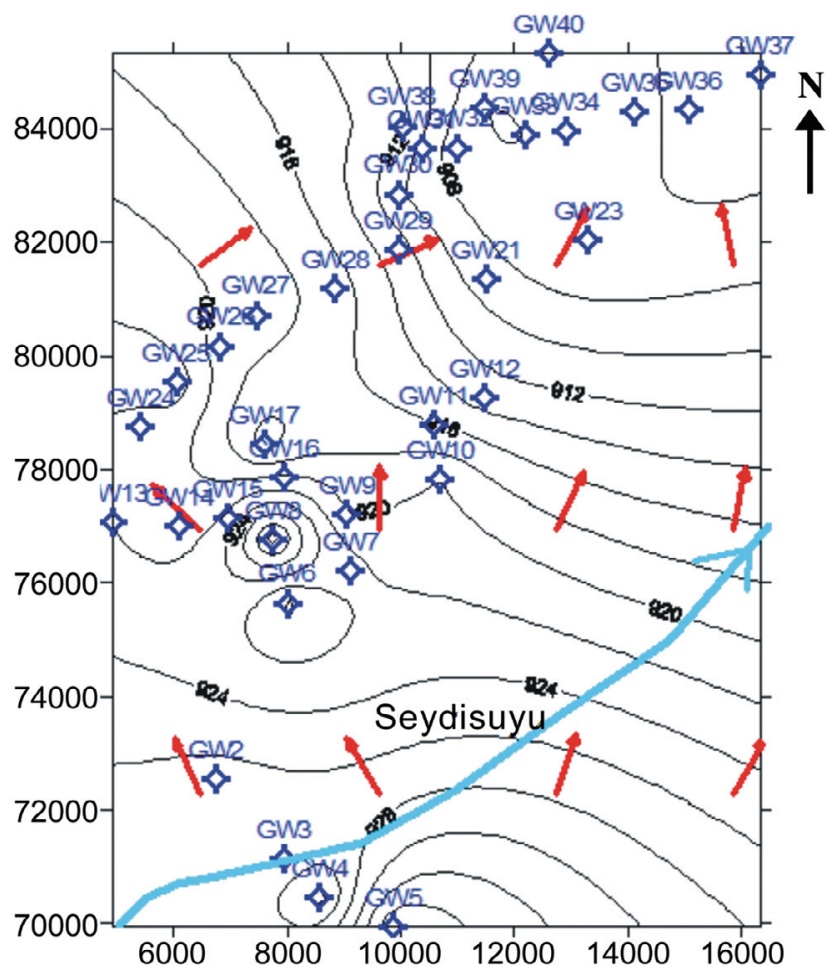

Fig. 3. Groundwater equipotential lines (modified from Ugurluoglu 2004; Ugurluoglu and Yuce 2004). 
to red being consistent with the increasing boron content is measured by spectrophotometer. The carmine method ( $\leq 0.05$ error limit) uses the complex of carmine (ananthraquinone dye) with boric acid in concentrated $\mathrm{H}_{2} \mathrm{SO}_{4}$ which has a $585 \mathrm{~nm}$ absorption maximum and $0.5-10 \mathrm{mg} \mathrm{l}^{-1}$ linear detection range. The direct (without matrix separation) determination of less than $0.5 \mathrm{mg} \mathrm{l}^{-1}$ by the carminic acid method is almost impossible in the presence of dissolved substances. The arsenic contents (2007) were carried out by ICP-MS in HU labs and its detection limit was near $0.5 \mu \mathrm{g} \mathrm{l}^{-1}$. Samples were filtered by $0.45 \mu$ and stabilized by ultrapure $\mathrm{HNO}_{3}$ for arsenic analysis. Both analytical results in the RASE and HU Laboratories were consistent with each other.

Stable isotopes, $\delta^{18} \mathrm{O}$ ve $\delta^{2} \mathrm{H}$, were analyzed using a thermo-chemical elemental analyzer (TCEA) coupled to a Thermo Finnigan Delta Plus XL Isotope Ratio Mass Spectrometer through an open split interface at laboratories in Stable Isotope Ratio Facility for Environmental Research
Department of Biology of the University of Utah (USA). Limits of precision were \pm 1 for $\delta^{2} \mathrm{H}$ and \pm 0.2 for $\delta^{18} \mathrm{O}$. Tritium was analyzed in the laboratory at Hacettepe University with a liquid scintillation counter (LSC) after electrolytic enrichment of the water samples with an error of \pm 0.8 Tritium Units Collected isotope samples were placed in a cold environment to eliminate evaporation effect till analyzing.

\section{RESULTS AND DISCUSSION}

\subsection{Groundwater}

A total of 136 groundwater samples were collected from the 42 deep bores drilled in the Seyitgazi Plain. The analytical results of groundwater samples performed between 1971 and 2003 and relatively recent (2007) were given in Table 1, respectively. The minimum, maximum and average concentrations are presented in Table 2. The boron concentration varies from below the detection limit to $7.82 \mathrm{ppm}$.

Table 1. Analytical results of groundwater samples.

\begin{tabular}{|c|c|c|c|c|c|c|c|c|c|c|c|c|c|c|c|c|c|}
\hline No & $\begin{array}{c}\text { Sample } \\
\text { Date }\end{array}$ & pH & $\begin{array}{c}\mathrm{EC} \\
\mathrm{mho} / \mathrm{cm}\end{array}$ & $\mathbf{N a}^{+}$ & $\mathbf{K}^{+}$ & $\mathrm{Ca}^{++}$ & $\mathbf{M g}^{++}$ & $\mathrm{CO}_{3}^{--}$ & $\mathrm{HCO}_{3}^{-}$ & $\mathrm{Cl}^{-}$ & $\mathrm{SO}_{4}^{--}$ & $\begin{array}{c}\text { B } \\
(\mathbf{p p m})\end{array}$ & As & $\mathrm{Sr}$ & $\delta^{18} \mathbf{O}$ & $\delta \mathrm{D}$ & $\mathbf{T}$ \\
\hline GW1 & $1990 \mathrm{~W}$ & & & & & & & & & & & 0.03 & & & & & \\
\hline GW1 & 2003 D & 7.90 & 327 & 11.9 & 5.7 & 31.7 & 17.2 & 16.0 & 152.6 & 18.3 & 5.4 & 0.66 & & & & & \\
\hline GW2 & $1990 \mathrm{D}$ & & & & & & & & & & & 0.17 & & & & & \\
\hline GW2 & 1999 D & & & & & & & & & & & 0.43 & 0.013 & & & & \\
\hline GW2 & 2003 D & 7.98 & 323 & 17.5 & 7.0 & 32.1 & 17.0 & 15.0 & 166.3 & 18.6 & 9.1 & 0.52 & & & & & \\
\hline GW2 & $2007 \mathrm{~W}$ & 7.37 & 418 & 12.3 & 4.2 & 49.2 & 21.0 & 0.0 & 265.8 & 5.8 & 11.0 & 0.08 & 0.008 & 0.54 & -8.34 & -71.2 & 1.87 \\
\hline GW2 & $2007 \mathrm{D}$ & 7.37 & 451 & 14.6 & 5.8 & 40.3 & 19.4 & 0.0 & 235.8 & 8.1 & 18.0 & 0.13 & 0.020 & 0.68 & -9.48 & -71.5 & 0.49 \\
\hline GW3 & $1990 \mathrm{D}$ & & & & & & & & & & & 0.39 & 0.010 & & & & \\
\hline GW3 & $2000 \mathrm{D}$ & & & & & & & & & & & 0.11 & & & & & \\
\hline GW3 & 2003 D & 8.05 & 355 & 15.2 & 6.5 & 39.6 & 17.6 & 12.0 & 189.2 & 19.5 & 9.6 & 0.54 & & & & & \\
\hline GW4 & $1990 \mathrm{D}$ & & & & & & & & & & & 0.11 & & & & & \\
\hline GW4 & $1999 \mathrm{D}$ & & & & & & & & & & & 0.17 & 0.010 & & & & \\
\hline GW4 & $2001 \mathrm{D}$ & & & & & & & & & & & 0.10 & & & & & \\
\hline GW4 & 2003 D & 8.03 & 440 & 21.9 & 5.7 & 42.1 & 25.5 & 8.0 & 205.4 & 27.2 & 43.2 & 0.46 & & & & & \\
\hline GW5 & $1990 \mathrm{D}$ & & & & & & & & & & & 0.11 & & & & & \\
\hline GW5 & $1999 \mathrm{D}$ & & & & & & & & & & & 0.14 & & & & & \\
\hline GW5 & $2001 \mathrm{D}$ & & & & & & & & & & & 0.10 & & & & & \\
\hline GW5 & 2003 D & 8.00 & 605 & 35.4 & 3.5 & 88.2 & 18.2 & 6.0 & 292.9 & 33.7 & 75.9 & 0.53 & & & & & \\
\hline GW6 & 1997 D & & & & & & & & & & & 0.78 & & & & & \\
\hline GW6 & $2003 \mathrm{D}$ & 7.70 & 465 & 19.0 & 5.7 & 56.1 & 23.4 & 6.0 & 244.1 & 27.5 & 35.8 & 0.89 & & & & & \\
\hline GW7 & $1997 \mathrm{~W}$ & & & & & & & & & & & 0.69 & & & & & \\
\hline GW7 & 1999 D & & & & & & & & & & & 0.68 & 0.013 & & & & \\
\hline GW7 & $2000 \mathrm{D}$ & & & & & & & & & & & 0.50 & & & & & \\
\hline GW7 & 2003 D & 7.83 & 397 & 15.0 & 5.5 & 45.8 & 22.5 & 10.0 & 199.3 & 21.3 & 34.9 & 0.96 & & & & & \\
\hline GW8 & $1997 \mathrm{~W}$ & & & & & & & & & & & 0.98 & & & & & \\
\hline GW8 & 2003 D & 8.00 & 430 & 46.8 & 4.5 & 41.1 & 13.4 & 9.0 & 219.7 & 23.0 & 36.0 & 1.23 & & & & & \\
\hline GW9 & $1997 \mathrm{~W}$ & & & & & & & & & & & 0.92 & & & & & \\
\hline GW9 & 2003 D & 7.80 & 830 & 90.1 & 4.3 & 84.2 & 23.4 & 6.0 & 302.0 & 34.6 & 193.5 & 1.80 & & & & & \\
\hline GW10 & 1997 W & & & & & & & & & & & 1.60 & & & & & \\
\hline GW10 & 2003 D & 7.75 & 660 & 60.1 & 4.5 & 73.1 & 21.9 & 6.0 & 329.5 & 26.6 & 88.0 & 1.18 & & & & & \\
\hline
\end{tabular}


Table 1. (Continued)

\begin{tabular}{|c|c|c|c|c|c|c|c|c|c|c|c|c|c|c|c|c|c|}
\hline No & $\begin{array}{c}\text { Sample } \\
\text { Date }\end{array}$ & $\mathbf{p H}$ & $\begin{array}{c}\text { EC } \\
\text { mho/cm }\end{array}$ & $\mathrm{Na}^{+}$ & $\mathbf{K}^{+}$ & $\mathrm{Ca}^{++}$ & $\mathbf{M g}^{++}$ & $\mathrm{CO}_{3}^{--}$ & $\mathrm{HCO}_{3}^{-}$ & $\mathrm{Cl}^{-}$ & $\mathrm{SO}_{4}^{--}$ & $\begin{array}{c}\mathbf{B} \\
(\mathbf{p p m})\end{array}$ & As & $\mathrm{Sr}$ & $\delta^{18} O$ & $\delta \mathrm{D}$ & $\mathbf{T}$ \\
\hline GW11 & 1997 D & & & & & & & & & & & 0.89 & & & & & \\
\hline GW11 & $2001 \mathrm{D}$ & 7.70 & 630 & 25.3 & 5.5 & 40.1 & 52.3 & 12.0 & 231.9 & 26.6 & 124.0 & 0.06 & & & & & \\
\hline GW11 & 2003 D & 7.78 & 678 & 72.3 & 5.8 & 55.4 & 26.9 & 9.0 & 267.0 & 33.2 & 127.3 & 0.76 & & & & & \\
\hline GW12 & 1997 D & & & & & & & & & & & 1.37 & 0.028 & & & & \\
\hline GW12 & 1999 D & & & & & & & & & & & 1.86 & & & & & \\
\hline GW12 & $2000 \mathrm{D}$ & & & & & & & & & & & 1.74 & & & & & \\
\hline GW12 & $2001 \mathrm{D}$ & 7.75 & 785 & 52.7 & 5.7 & 65.6 & 21.9 & 6.0 & 134.4 & 44.3 & 185.4 & 0.68 & & & & & \\
\hline GW12 & 2003 D & 7.93 & 670 & 90.0 & 4.8 & 53.1 & 17.8 & 10.0 & 268.5 & 39.6 & 110.6 & 1.77 & & & & & \\
\hline GW13 & $1990 \mathrm{~W}$ & & & & & & & & & & & 1.00 & & & & & \\
\hline GW13 & $2001 \mathrm{D}$ & 7.70 & 630 & 29.0 & 6.7 & 40.1 & 29.2 & 0.0 & 286.8 & 19.5 & 27.9 & 0.00 & & & & & \\
\hline GW14 & $1990 \mathrm{~W}$ & & & & & & & & & & & 1.37 & & & & & \\
\hline GW14 & $2007 \mathrm{~W}$ & 7.40 & 863 & 75.7 & 4.3 & 69.8 & 29.3 & 0.0 & 294.2 & 12.1 & 183.1 & 1.43 & 0.024 & 0.80 & -9.56 & -66.4 & 1.82 \\
\hline GW14 & 2007 D & 7.26 & 714 & 42.4 & 4.8 & 56.2 & 24.4 & 0.0 & 286.0 & 11.4 & 103.8 & 1.35 & 0.020 & 0.79 & -9.02 & -66.9 & 0.91 \\
\hline GW15 & $1990 \mathrm{~W}$ & & & & & & & & & & & 1.64 & & & & & \\
\hline GW15 & 1999 D & & & & & & & & & & & 1.70 & 0.017 & & & & \\
\hline GW15 & 2003 D & 7.83 & 413 & 40.7 & 4.1 & 42.3 & 13.7 & 7.5 & 233.4 & 19.9 & 22.8 & 1.93 & & & & & \\
\hline GW16 & $1990 \mathrm{~W}$ & & & & & & & & & & & 1.60 & & & & & \\
\hline GW16 & $2001 \mathrm{D}$ & 7.70 & 680 & 43.0 & 5.9 & 60.1 & 28.6 & 0.0 & 292.9 & 28.4 & 85.0 & 0.00 & & & & & \\
\hline GW16 & 2003 D & 7.85 & 485 & 31.4 & 4.7 & 60.1 & 18.5 & 6.0 & 238.0 & 23.0 & 60.5 & 1.31 & & & & & \\
\hline GW17 & $1990 \mathrm{~W}$ & & & & & & & & & & & 0.90 & & & & & \\
\hline GW17 & $2001 \mathrm{D}$ & 7.80 & 570 & 34.7 & 6.3 & 69.1 & 24.3 & 0.0 & 311.2 & 30.1 & 56.2 & 0.00 & & & & & \\
\hline GW17 & 2003 D & 7.70 & 447 & 26.7 & 5.7 & 57.8 & 16.6 & 8.0 & 252.2 & 23.6 & 23.5 & 1.66 & & & & & \\
\hline GW18 & $1990 \mathrm{~W}$ & & & & & & & & & & & 0.76 & & & & & \\
\hline GW18 & 1999 D & & & & & & & & & & & 2.94 & 0.016 & & & & \\
\hline GW18 & $2001 \mathrm{D}$ & 7.70 & 695 & 37.1 & 7.0 & 63.1 & 35.2 & 0.0 & 137.3 & 27.5 & 226.5 & 0.48 & & & & & \\
\hline GW18 & 2003 D & 7.50 & 540 & 26.9 & 8.6 & 74.2 & 19.4 & 6.0 & 201.4 & 21.3 & 124.0 & 1.21 & & & & & \\
\hline GW18 & $2007 \mathrm{~W}$ & 7.27 & 674 & 28.3 & 7.1 & 73.1 & 27.3 & 0.0 & 253.9 & 8.6 & 120.4 & 0.83 & 0.014 & 0.80 & -8.66 & -69.6 & 0.19 \\
\hline GW18 & 2007 D & 7.31 & 707 & 24.6 & 6.1 & 68.4 & 25.6 & 0.0 & 261.0 & 9.4 & 135.1 & 0.88 & 0.020 & 0.89 & -9.53 & -68.7 & 0.29 \\
\hline GW19 & $1990 \mathrm{~W}$ & & & & & & & & & & & 0.50 & & & & & \\
\hline GW19 & 1999 D & & & & & & & & & & & 4.30 & 0.026 & & & & \\
\hline GW19 & $2001 \mathrm{D}$ & 7.80 & 560 & 16.3 & 5.1 & 52.1 & 29.8 & 6.0 & 305.1 & 17.7 & 9.1 & 0.00 & & & & & \\
\hline GW19 & 2003 D & 7.73 & 838 & 95.7 & 6.1 & 70.1 & 22.5 & 10.5 & 344.8 & 27.0 & 139.6 & 1.40 & & & & & \\
\hline GW20 & $2001 \mathrm{D}$ & 7.65 & 735 & 26.7 & 7.0 & 73.1 & 31.3 & 6.0 & 146.5 & 26.6 & 202.3 & 0.00 & & & & & \\
\hline GW20 & 2003 D & 7.70 & 655 & 41.4 & 8.9 & 80.9 & 22.2 & 6.0 & 288.3 & 23.9 & 110.2 & 1.41 & & & & & \\
\hline GW21 & $1990 \mathrm{~W}$ & & & & & & & & & & & 0.95 & & & & & \\
\hline GW21 & $2001 \mathrm{D}$ & 7.75 & 690 & 23.7 & 8.4 & 73.7 & 19.1 & 3.0 & 140.4 & 23.9 & 159.3 & 0.51 & & & & & \\
\hline GW21 & 2003 D & 7.65 & 610 & 35.7 & 9.4 & 77.2 & 22.6 & 6.0 & 233.4 & 26.1 & 131.5 & 1.23 & & & & & \\
\hline GW22 & 1990 D & & & & & & & & & & & 0.40 & & & & & \\
\hline GW22 & 1999 D & & & & & & & & & & & 4.30 & 0.018 & & & & \\
\hline GW22 & $2001 \mathrm{D}$ & 7.70 & 735 & 25.9 & 10.0 & 67.1 & 29.5 & 6.0 & 149.5 & 24.8 & 183.0 & 0.41 & & & & & \\
\hline GW22 & 2003 D & 7.40 & 517 & 32.3 & 8.9 & 64.5 & 18.8 & 6.0 & 229.8 & 24.8 & 83.1 & 1.04 & & & & & \\
\hline GW22 & $2007 \mathrm{~W}$ & 7.35 & 617 & 27.2 & 7.4 & 66.9 & 21.1 & 0.0 & 229.1 & 9.5 & 102.5 & 0.51 & 0.016 & 0.68 & -9.21 & -71.5 & 0.54 \\
\hline GW22 & 2007 D & 7.33 & 628 & 24.9 & 7.4 & 60.1 & 19.5 & 0.0 & 220.1 & 11.7 & 109.3 & 0.55 & 0.020 & 0.70 & -9.78 & -74 & 0.28 \\
\hline GW23 & 1997 D & & & & & & & & & & & 0.36 & & & & & \\
\hline GW23 & 1999 D & & & & & & & & & & & 2.66 & 0.015 & & & & \\
\hline GW23 & $2000 \mathrm{D}$ & & & & & & & & & & & 0.45 & & & & & \\
\hline GW23 & $2001 \mathrm{D}$ & 7.70 & 530 & 19.5 & 4.7 & 70.1 & 23.1 & 12.0 & 170.9 & 24.8 & 119.0 & 0.08 & & & & & \\
\hline GW23 & 2003 D & 7.80 & 470 & 41.6 & 8.0 & 44.6 & 17.0 & 9.0 & 219.7 & 36.3 & 34.4 & 0.79 & & & & & \\
\hline GW24 & $2001 \mathrm{D}$ & 7.83 & 657 & 34.6 & 7.0 & 68.5 & 27.1 & 10.0 & 250.2 & 40.2 & 84.9 & 0.57 & & & & & \\
\hline GW25 & $1989 \mathrm{~W}$ & & & & & & & & & & & 0.42 & & & & & \\
\hline GW25 & $2001 \mathrm{D}$ & 7.80 & 673 & 24.0 & 6.1 & 63.5 & 38.1 & 10.0 & 262.4 & 32.5 & 93.6 & 0.49 & & & & & \\
\hline GW26 & $2001 \mathrm{D}$ & 7.90 & 635 & 30.1 & 5.9 & 64.6 & 31.9 & 9.0 & 262.4 & 32.8 & 85.6 & 0.29 & & & & & \\
\hline GW26 & 2003 D & 7.53 & 608 & 41.4 & 6.8 & 68.6 & 25.2 & 9.0 & 219.7 & 30.6 & 130.3 & 1.58 & & & & & \\
\hline
\end{tabular}


Table 1. (Continued)

\begin{tabular}{|c|c|c|c|c|c|c|c|c|c|c|c|c|c|c|c|c|c|}
\hline No & $\begin{array}{c}\text { Sample } \\
\text { Date }\end{array}$ & $\mathbf{p H}$ & $\begin{array}{c}\text { EC } \\
\text { mho/cm }\end{array}$ & $\mathbf{N a}^{+}$ & $\mathbf{K}^{+}$ & $\mathrm{Ca}^{++}$ & $\mathbf{M g}^{++}$ & $\mathrm{CO}_{3}^{--}$ & $\mathrm{HCO}_{3}^{-}$ & $\mathrm{Cl}^{-}$ & $\mathrm{SO}_{4}^{--}$ & $\begin{array}{c}\text { B } \\
(\mathbf{p p m})\end{array}$ & As & $\mathbf{S r}$ & $\delta^{18} \mathbf{O}$ & $\delta \mathrm{D}$ & $\mathbf{T}$ \\
\hline GW27 & $2001 \mathrm{D}$ & 7.83 & 563 & 19.2 & 4.8 & 62.5 & 26.1 & 8.0 & 219.7 & 35.5 & 65.2 & 0.50 & & & & & \\
\hline GW27 & 2003 D & 7.53 & 730 & 38.3 & 6.2 & 94.7 & 30.1 & 9.0 & 299.0 & 29.7 & 143.3 & 1.35 & & & & & \\
\hline GW28 & $1990 \mathrm{~W}$ & & & & & & & & & & & 1.00 & & & & & \\
\hline GW28 & $2001 \mathrm{D}$ & 7.83 & 670 & 34.1 & 7.0 & 68.8 & 32.8 & 8.0 & 252.2 & 35.5 & 115.0 & 0.38 & & & & & \\
\hline GW28 & 2003 D & 7.90 & 820 & 104.1 & 6.4 & 70.6 & 23.2 & 12.0 & 347.8 & 23.9 & 161.1 & 1.24 & & & & & \\
\hline GW29 & 1999 D & & & & & & & & & & & 0.81 & 0.010 & & & & \\
\hline GW29 & $2001 \mathrm{D}$ & 7.78 & 713 & 22.6 & 8.2 & 80.7 & 34.3 & 7.5 & 192.2 & 44.8 & 162.3 & 0.24 & & & & & \\
\hline GW29 & 2003 D & 7.65 & 855 & 59.7 & 8.5 & 100.4 & 32.2 & 10.5 & 331.0 & 46.5 & 162.8 & 1.17 & & & & & \\
\hline GW30 & $2001 \mathrm{D}$ & 7.80 & 703 & 21.8 & 6.5 & 79.2 & 25.5 & 6.0 & 244.1 & 39.9 & 130.5 & 0.38 & & & & & \\
\hline GW30 & 2003 D & 7.78 & 758 & 62.5 & 7.2 & 85.2 & 27.1 & 9.0 & 312.7 & 32.3 & 146.8 & 1.22 & & & & & \\
\hline GW31 & 1989 D & & & & & & & & & & & 0.25 & & & & & \\
\hline GW31 & $2001 \mathrm{D}$ & 7.77 & 650 & 23.7 & 5.9 & 78.5 & 25.5 & 6.0 & 260.4 & 39.0 & 78.4 & 0.02 & & & & & \\
\hline GW31 & 2003 D & 7.93 & 495 & 23.5 & 8.0 & 54.9 & 26.4 & 9.0 & 227.3 & 32.4 & 57.7 & 0.81 & & & & & \\
\hline GW32 & 1989 D & & & & & & & & & & & 0.50 & & & & & \\
\hline GW32 & $2002 \mathrm{~W}$ & 7.78 & 638 & 25.6 & 7.5 & 65.1 & 32.8 & 6.0 & 199.8 & 28.4 & 143.0 & 0.22 & & & & & \\
\hline GW32 & 2003 D & 7.85 & 510 & 25.8 & 8.4 & 65.1 & 21.0 & 12.0 & 204.4 & 23.9 & 90.7 & 0.71 & & & & & \\
\hline GW33 & $2001 \mathrm{D}$ & 7.80 & 673 & 23.9 & 8.3 & 74.5 & 27.3 & 8.0 & 240.0 & 35.5 & 96.9 & 0.38 & & & & & \\
\hline GW33 & 2003 D & 7.60 & 553 & 28.2 & 9.3 & 58.8 & 29.8 & 10.0 & 229.8 & 27.8 & 94.4 & 1.21 & & & & & \\
\hline GW34 & 1997 D & & & & & & & & & & & 0.73 & & & & & \\
\hline GW34 & 1999 D & 7.80 & 700 & 17.5 & 9.0 & 74.2 & 29.2 & 6.0 & 231.9 & 33.7 & 103.0 & 1.86 & 0.013 & & & & \\
\hline GW34 & $2000 \mathrm{D}$ & & & & & & & & & & & 0.31 & & & & & \\
\hline GW34 & $2001 \mathrm{D}$ & 7.70 & 665 & 21.2 & 6.5 & 74.2 & 34.6 & 9.0 & 250.2 & 38.1 & 104.0 & 0.28 & & & & & \\
\hline GW34 & 2003 D & 7.93 & 537 & 30.1 & 8.1 & 64.8 & 21.3 & 10.0 & 219.7 & 34.9 & 76.1 & 1.19 & & & & & \\
\hline GW35 & 1997 W & & & & & & & & & & & 0.62 & & & & & \\
\hline GW35 & $2001 \mathrm{D}$ & 7.70 & 753 & 26.3 & 9.1 & 78.7 & 34.8 & 4.5 & 181.5 & 34.1 & 196.0 & 0.41 & & & & & \\
\hline GW35 & 2003 D & 7.78 & 583 & 23.7 & 9.1 & 75.7 & 25.1 & 12.0 & 231.9 & 32.4 & 95.4 & 1.20 & & & & & \\
\hline GW36 & 1997 D & & & & & & & & & & & 0.57 & & & & & \\
\hline GW36 & 1999 D & & & & & & & & & & & 0.42 & & & & & \\
\hline GW36 & $2001 \mathrm{D}$ & 7.77 & 600 & 21.7 & 6.1 & 58.8 & 31.2 & 8.0 & 225.8 & 31.3 & 84.0 & 0.54 & & & & & \\
\hline GW36 & 2003 D & 7.73 & 525 & 26.2 & 7.2 & 61.6 & 24.3 & 13.5 & 251.7 & 31.0 & 45.6 & 1.40 & & & & & \\
\hline GW37 & $1997 \mathrm{~W}$ & & & & & & & & & & & 0.41 & & & & & \\
\hline GW37 & 1999 D & & & & & & & & & & & 1.46 & 0.011 & & & & \\
\hline GW37 & $2001 \mathrm{D}$ & 7.63 & 597 & 24.8 & 6.1 & 56.1 & 30.8 & 4.0 & 250.2 & 25.4 & 77.5 & 0.29 & & & & & \\
\hline GW37 & 2003 D & 7.73 & 420 & 21.2 & 5.4 & 46.3 & 21.7 & 21.0 & 209.0 & 28.8 & 10.7 & 1.08 & & & & & \\
\hline GW38 & $1997 \mathrm{~W}$ & & & & & & & & & & & 0.19 & & & & & \\
\hline GW38 & 1999 D & & & & & & & & & & & 0.50 & 0.017 & & & & \\
\hline GW38 & $2001 \mathrm{D}$ & 7.77 & 593 & 29.9 & 8.3 & 57.1 & 21.5 & 4.0 & 240.0 & 33.1 & 54.3 & 0.19 & & & & & \\
\hline GW38 & 2003 D & 8.00 & 505 & 31.3 & 5.1 & 56.1 & 19.9 & 16.5 & 207.5 & 30.6 & 53.6 & 1.21 & & & & & \\
\hline GW39 & 1997 D & & & & & & & & & & & 0.29 & & & & & \\
\hline GW39 & 1999 D & & & & & & & & & & & 0.36 & 0.011 & & & & \\
\hline GW39 & $2001 \mathrm{D}$ & 8.10 & 515 & 19.8 & 6.5 & 61.6 & 19.7 & 18.0 & 201.4 & 26.6 & 51.6 & 0.09 & & & & & \\
\hline GW39 & 2003 D & 7.85 & 483 & 25.2 & 6.4 & 58.1 & 21.0 & 12.0 & 187.6 & 35.5 & 67.7 & 0.89 & & & & & \\
\hline GW40 & 1997 D & & & & & & & & & & & 0.35 & & & & & \\
\hline GW40 & 1999 D & & & & & & & & & & & 0.50 & 0.012 & & & & \\
\hline GW40 & $2001 \mathrm{D}$ & 8.15 & 525 & 23.3 & 7.0 & 59.1 & 20.7 & 15.0 & 198.3 & 23.9 & 69.6 & 0.02 & & & & & \\
\hline GW40 & 2003 D & 7.85 & 485 & 25.9 & 7.2 & 56.4 & 21.7 & 7.5 & 190.7 & 32.4 & 78.2 & 0.96 & & & & & \\
\hline GW41 & $2002 \mathrm{~W}$ & 7.90 & 460 & 7.6 & 3.1 & 61.1 & 24.9 & 24.0 & 244.0 & 7.1 & 24.5 & 3.95 & & & & & \\
\hline GW41 & $2003 \mathrm{~W}$ & 7.92 & 370 & 10.8 & 3.2 & 41.9 & 23.3 & 7.2 & 229.4 & 15.6 & 5.8 & 6.49 & & & & & \\
\hline GW41 & 2003 D & 7.80 & 363 & 10.8 & 3.2 & 39.4 & 24.2 & 12.0 & 213.5 & 17.3 & 5.5 & 7.82 & & & & & \\
\hline GW41 & $2007 \mathrm{~W}$ & 7.28 & 428 & 10.7 & 2.5 & 45.5 & 27.0 & 0.0 & 278.7 & 3.3 & 6.2 & 6.32 & 0.028 & 3.16 & -9.44 & -68.7 & 0.8 \\
\hline GW41 & 2007 D & 7.41 & 435 & 9.3 & 2.5 & 52.6 & 26.6 & 0.0 & 273.6 & 6.9 & 14.1 & 6.26 & 0.030 & 3.61 & -9.98 & -69.6 & 0.37 \\
\hline GW42 & 1988 D & & & & & & & & & & & 6.90 & & & & & \\
\hline GW42 & $1989 \mathrm{~W}$ & & & & & & & & & & & 7.50 & & & & & \\
\hline
\end{tabular}


Table 2. The results of the minimum, maximum and average concentrations of boron values in groundwater.

\begin{tabular}{|c|c|c|c|}
\hline \multirow[b]{2}{*}{ Sample No } & \multicolumn{3}{|c|}{ Boron (ppm) } \\
\hline & Average & Minimum & Maximum \\
\hline GW1 & 0.34 & 0.03 & 0.66 \\
\hline GW2 & 0.31 & 0.08 & 0.52 \\
\hline GW3 & 0.49 & 0.39 & 0.54 \\
\hline GW4 & 0.21 & 0.10 & 0.46 \\
\hline GW5 & 0.22 & 0.10 & 0.53 \\
\hline GW6 & 0.83 & 0.78 & 0.89 \\
\hline GW7 & 0.71 & 0.50 & 0.96 \\
\hline GW8 & 1.10 & 0.98 & 1.23 \\
\hline GW9 & 1.36 & 0.92 & 1.80 \\
\hline GW10 & 1.39 & 1.18 & 1.60 \\
\hline GW11 & 0.57 & 0.06 & 0.89 \\
\hline GW12 & 1.48 & 0.68 & 1.86 \\
\hline GW13 & 0.50 & 0.00 & 1.00 \\
\hline GW14 & 1.38 & 1.35 & 1.43 \\
\hline GW15 & 1.76 & 1.64 & 1.93 \\
\hline GW16 & 0.97 & 0.00 & 1.60 \\
\hline GW17 & 0.85 & 0.00 & 1.66 \\
\hline GW18 & 1.18 & 0.48 & 2.94 \\
\hline GW19 & 1.55 & 0.50 & 4.30 \\
\hline GW21 & 0.90 & 0.51 & 1.23 \\
\hline GW22 & 1.54 & 0.40 & 1.04 \\
\hline GW22 & 0.53 & 0.51 & 0.55 \\
\hline GW23 & 0.41 & 0.08 & 0.79 \\
\hline GW24 & 0.57 & 0.57 & 0.57 \\
\hline GW25 & 0.45 & 0.42 & 0.49 \\
\hline GW26 & 0.93 & 0.29 & 1.58 \\
\hline GW27 & 0.93 & 0.50 & 1.35 \\
\hline GW28 & 0.87 & 0.38 & 1.24 \\
\hline GW29 & 0.74 & 0.24 & 1.17 \\
\hline GW30 & 0.80 & 0.38 & 1.22 \\
\hline GW31 & 0.36 & 0.02 & 0.81 \\
\hline GW32 & 0.48 & 0.22 & 0.71 \\
\hline GW33 & 0.79 & 0.38 & 1.21 \\
\hline GW34 & 0.87 & 0.28 & 1.86 \\
\hline GW36 & 0.73 & 0.42 & 1.40 \\
\hline GW37 & 0.81 & 0.29 & 1.46 \\
\hline GW38 & 0.52 & 0.50 & 1.21 \\
\hline GW39 & 0.41 & 0.09 & 0.89 \\
\hline GW40 & 0.46 & 0.02 & 0.96 \\
\hline GW41 & 6.09 & 3.95 & 7.82 \\
\hline GW42 & 6.91 & 6.90 & 7.50 \\
\hline
\end{tabular}

Increase in $\mathrm{pH}$ value of groundwater may facilitate the mobilization of boron, locally (Demirtas 2006). Multiple correlation analysis was computed by Excel and the relationships between ions of groundwater samples were denoted on the wet (Tables $3 \mathrm{a}$ and $\mathrm{b}$ ) and the dry periods (Tables $3 \mathrm{c}$ and d). Boron shows a strong positive correlation with sodium in groundwater.

The dissolution of sulfate mineral increased during the wet period. Concordantly, the correlation coefficients ( $r$ ) of $\mathrm{SO}_{4}$ with $\mathrm{Ca}$ and $\mathrm{Mg}$ were 0.84 and 0.91 for the wet periods. The correlation coefficients between $\mathrm{Na}$ and $\mathrm{B}$ in the wet season were eventually higher $(r=0.92)$ than the dry season $(r<0.50)$ as a result of increasing solubility by rainfall. Based upon all of analytical results of groundwater samples showed that boron concentration has increased since 1989 (Fig. 4); in particular, the years between 1990 and 1999, B concentrations increased 2.5 to 10 times higher from the initial concentrations in groundwater (except for GW2, GW14 GW22) at the Seyitgazi Plain. The arsenic concentration also increased in groundwater which exceeded the allowable limit of $10 \mu \mathrm{g} \mathrm{l}^{-1}$ (EPA 2001) in the 14 wells out of 16 (Table 1). There is a strong correlation between arsenic, strontium and boron concentrations both in the wet and dry periods (Tables $3 \mathrm{~b}$ and d). Arsenic concentrations are $25 \mu \mathrm{g} \mathrm{l}^{-1}$ in the Seydisuyu stream, $26 \mu \mathrm{g} \mathrm{l}^{-1}$ in the public water-supply system and $30 \mu \mathrm{g} \mathrm{l}^{-1}$ in the well no. GW41 that are higher than the allowable limit for drinking purposes. The previous studies have also revealed that As and Sr concentrations in water resources around boron deposit areas are generally higher (Helvaci and Alonso 2000; Helvaci et al. 2004; Gemici et al. 2008; Zheng and Liu 2009).

A borax mine is located in the catchment area of the Catören and Kunduzlar dams. Boron is leached from the mine through rainwater and discharges into streams which recharge the lake at the Catören dam. Irrigation canals in the study area were constructed to provide irrigation water for the Seyitgazi Plain. Thus, water comes from Catören and Kunduzlar dams are conveyed via canals to the Seyitgazi Plain for irrigation. However, water high in boron content is infiltrated into aquifer system in the Seyitgazi Plain. Eventually, the boron level in groundwater in some parts of Seyitgazi Plain exceeds the recommended limit for irrigation purposes (10 $\mu \mathrm{g} \mathrm{l}^{-1}$, EPA 2001) due to percolation of the Catören reservoir's water into groundwater. For this reason, the boron concentration in groundwater of the Seyitgazi Plain has increased since 1989 and is still increasing in both catchment and disharge areas due to haphazard deposition of borate waste at the tailing of the mine site. As a result, most of the groundwater samples were over than the allowable boron limits for irrigation and drinking purposes according to the WHO, 2006. Particularly, the boron concentration in the wells, GW34, GW35, GW36, GW38, GW39 and GW40, increased up to three times, or even four higher than the initial concentration in consistence with the 
location of filtered shallow levels in the water wells (Fig. 5). However, boron concentrations in the wells, GW19 and GW20 which their filtered level were installed in relatively deeper depth, also increased in time due to the over-exploitation even. On the other hand, none of the deep wells at the Seyitgazi Plain were cut in boron bearing layers. Therefore, the high boron content of groundwater at the Seyitgazi Plain was sourced from irrigation water recharged by the Catören dam. As a result, boron increment in the groundwater in the Seyitgazi Plain was due to the infiltration of irrigation water provided from the Catören dam with a higher boron concentration.

Mineral equilibrium coefficients are a useful key for predicting the presence of reactive minerals and estimating mineral reactivity for future in a groundwater system. By using the saturation indices, one can easily predict the reactive minerals in host rocks, mineral dissolution and precipitation rates from the chemical analytical results of groundwater without examining on rock samples (Deutsch 1997). According to saturation index approach, groundwater was

Table 3a. Correlation values of groundwater (wet period) $(2002-2007)(n=7)$ (values less than 0.5 were omitted).

\begin{tabular}{|c|c|c|c|c|c|c|c|c|c|c|}
\hline & pH & EC & $\mathrm{Na}^{+}$ & $\mathbf{K}^{+}$ & $\mathrm{Ca}^{++}$ & $\mathbf{M g}^{++}$ & $\mathrm{CO}_{3}^{--}$ & $\mathrm{HCO}_{3}^{-}$ & $\mathrm{Cl}^{-}$ & $\mathrm{SO}_{4}^{--}$ \\
\hline $\mathbf{N a}^{+}$ & & 0.92 & & & & & & & & \\
\hline $\mathrm{Ca}^{++}$ & & 0.82 & 0.54 & 0.51 & & & & & & \\
\hline $\mathbf{M g}^{++}$ & 0.67 & 0.60 & & & 0.51 & & & & & \\
\hline $\mathrm{CO}_{3}^{--}$ & 0.97 & & & & & 0.70 & & & & \\
\hline $\mathrm{HCO}_{3}^{-}$ & & & 0.58 & & & & & & & \\
\hline $\mathrm{Cl}^{-}$ & 0.96 & & & & & 0.81 & 0.97 & & & \\
\hline $\mathrm{SO}_{4}^{--}$ & & 0.95 & 0.79 & & 0.84 & 0.76 & & & 0.50 & \\
\hline Bor & & 0.91 & 0.92 & & 0.69 & & & 0.65 & & 0.74 \\
\hline
\end{tabular}

Table 3b. Correlation values of groundwater (wet period) (2007) $(n=5)$ (values less than 0.5 were omitted).

\begin{tabular}{l|cccccccccc}
\hline & $\mathbf{p H}$ & $\mathbf{E C}$ & $\mathbf{N a}^{+}$ & $\mathbf{K}^{+}$ & $\mathbf{C a}^{++}$ & $\mathbf{M g}^{++}$ & $\mathbf{H C O}_{3}{ }^{-}$ & $\mathbf{C l}^{-}$ & Bor & $\mathbf{A s}$ \\
\hline $\mathbf{N a}^{+}$ & 0.58 & 0.94 & & & & & & & & \\
$\mathbf{C a}^{++}$ & & 0.87 & 0.65 & 0.77 & & & & & & \\
$\mathbf{M g}^{++}$ & & 0.57 & 0.58 & & & & & & & \\
$\mathbf{H C O}_{3}^{-}$ & & & & & & 0.70 & & & & \\
$\mathbf{C l}^{-}$ & 0.56 & 0.93 & 0.87 & 0.54 & 0.87 & & & & & \\
$\mathbf{S O}_{4}^{--}$ & & 0.99 & 0.90 & & 0.91 & & & 0.96 & & \\
$\mathbf{A s}$ & & & & & & 0.67 & 0.52 & & 0.86 & \\
$\mathbf{S r}$ & & & & & & & & & 0.99 & 0.80 \\
\hline
\end{tabular}

Table $3 \mathrm{c}$. Correlation values of groundwater (dry period) (2001 - 2007) $(\mathrm{n}=66)$ (values less than 0.5 were omitted).

\begin{tabular}{l|llll}
\hline & $\mathbf{p H}$ & $\mathbf{E C}$ & $\mathbf{N a}^{+}$ & $\mathbf{C a}^{++}$ \\
\hline $\mathrm{Na}^{+}$ & & 0.56 & & \\
$\mathbf{C a}^{++}$ & & 0.73 & & \\
$\mathrm{Mg}^{++}$ & & 0.50 & & \\
$\mathbf{C O}_{3}^{--}$ & 0.56 & & & \\
$\mathbf{H C O}_{3-}^{-}$ & & & 0.56 & \\
$\mathbf{S O}_{4--}^{--}$ & & 0.84 & & 0.68 \\
\hline
\end{tabular}

Table 3d. Correlation values of groundwater (dry period) $(2007)(n=5)$ (values less than 0.5 were omitted).

\begin{tabular}{l|ccccccccc}
\hline & $\mathbf{p H}$ & $\mathbf{E C}$ & $\mathbf{N a}^{+}$ & $\mathbf{K}^{+}$ & $\mathbf{C a}^{++}$ & $\mathbf{M g}^{++}$ & $\mathbf{H C O}_{3}{ }^{-}$ & $\mathbf{C l}$ & $\mathbf{B o r}$ \\
\hline $\mathbf{N a}^{+}$ & & 0.87 & & & & & & & \\
$\mathbf{C a}^{++}$ & & 0.77 & & & & & & & \\
$\mathbf{H C O}_{3}{ }^{-}$ & & & & & & 0.86 & & & \\
$\mathbf{C l}^{-}$ & & 0.81 & 0.84 & 0.66 & & & & & \\
$\mathbf{S O}_{4}^{--}$ & & 0.96 & 0.72 & 0.60 & 0.86 & & & 0.79 & \\
$\mathbf{B o r}$ & 0.59 & & & & & 0.68 & 0.51 & & \\
$\mathbf{A s}$ & 0.72 & & & & & 0.57 & & & 0.98 \\
$\mathbf{S r}$ & 0.69 & & & & & 0.62 & & & 0.99 \\
\hline
\end{tabular}



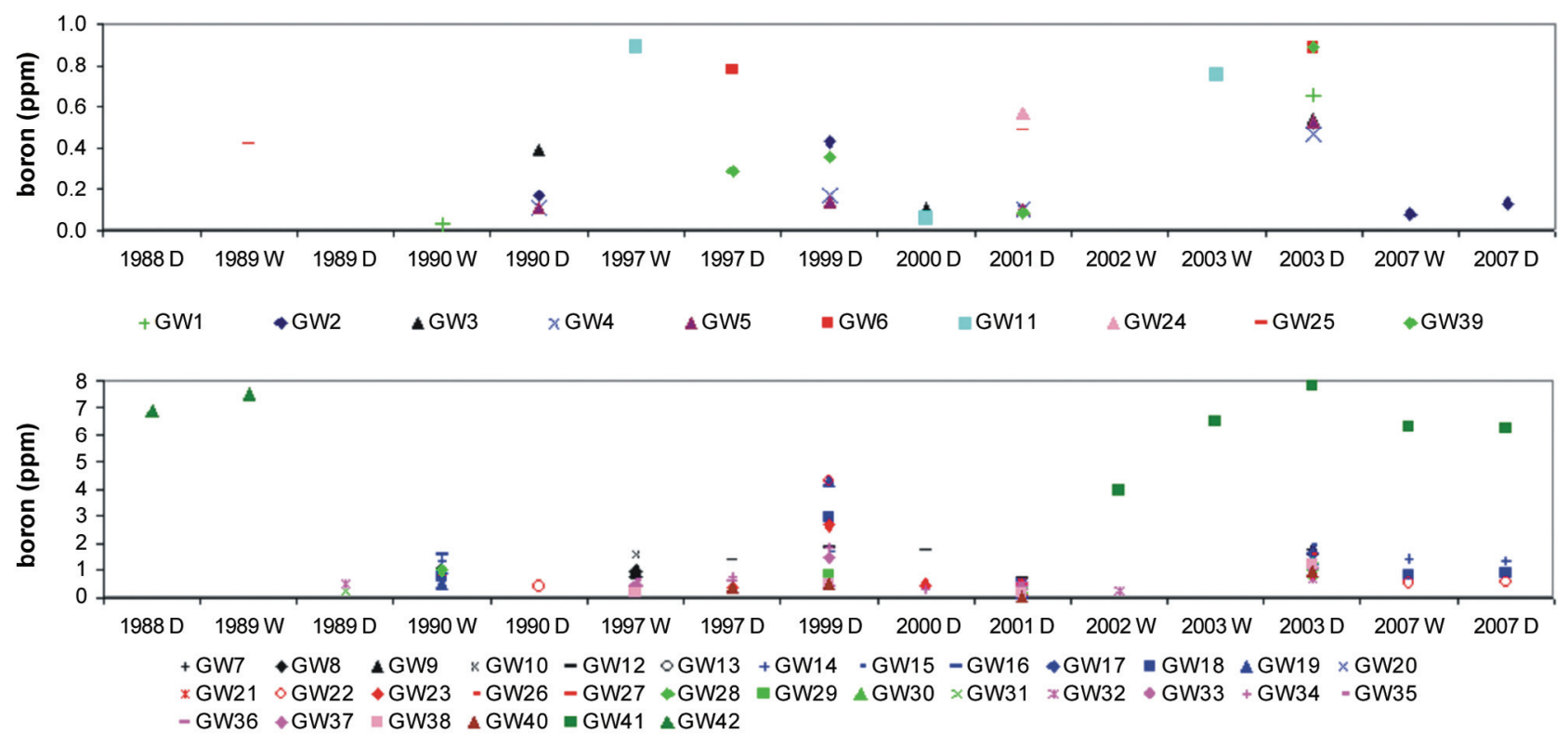

Fig. 4. Boron variations of groundwater in time.

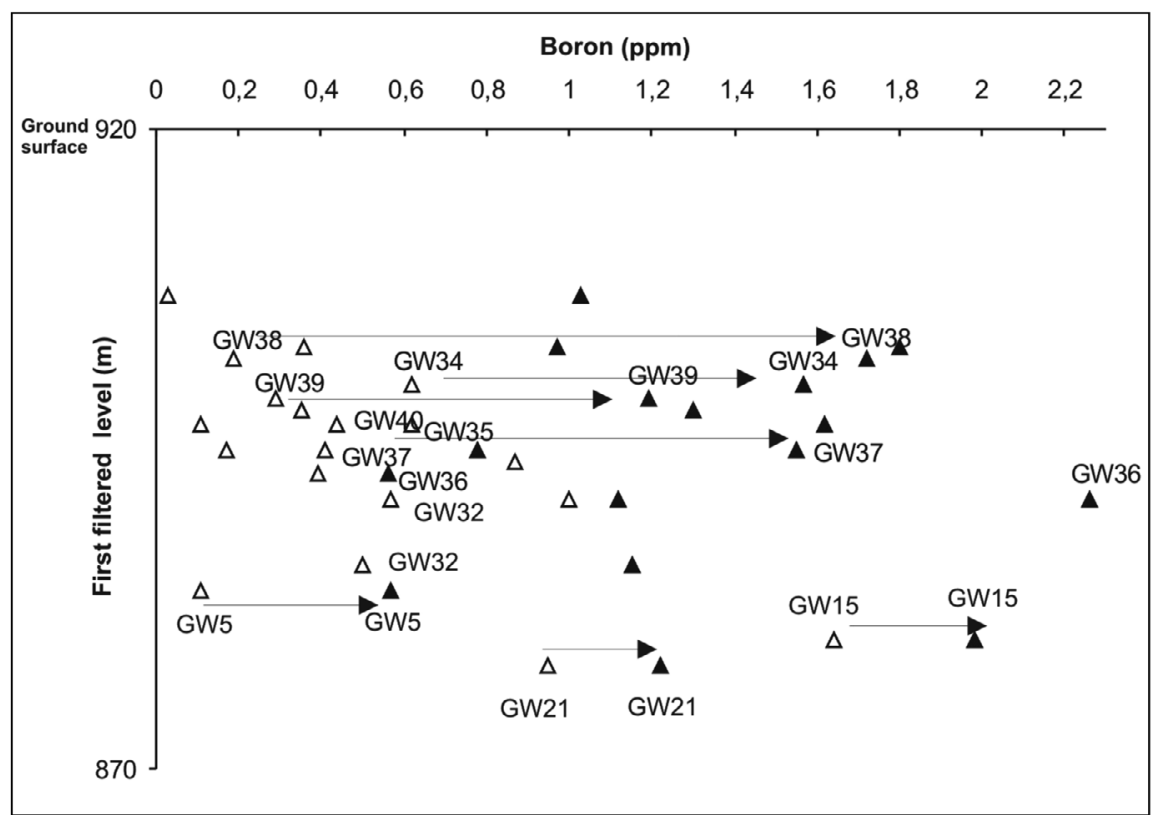

Fig. 5. Boron concentration as a function of time for first filtered level (modified from Ugurluoglu 2004). $\Delta$ : initial boron concentrations of groundwater when boreholes were drilled; $\mathbf{\Delta}$ : boron concentrations in 2003 (August/September); $\longrightarrow$ : relative boron increment rate.

almost supersaturated with respect to calcite and dolomite at the sampling temperatures (Fig. 6a). However, gypsum and anhydrite were under-saturated at the discharge temperature (Fig. 6b).

\subsection{Surface Water}

A total of 102 surface water samples were collected from the 15 different locations in the study area. Previous
(1971 - 2003) and relatively recent (2007) analytical results of surface water samples are given in Table 4 respectively. The minimum, maximum and average boron concentrations in surface waters are presented in Table 5. The minimum and maximum boron concentrations in the surface water ranged from 0.04 to $68 \mathrm{ppm}$. The highest boron concentration was found at location SW5 by $68 \mathrm{ppm}$. It is obvious that the boron concentration has increased since 1999 (Fig. 7). Paralleling the boron increase, the arsenic concen- 
tration in surface water was also higher than allowable limits for drinking water according to TS 266 (Table 4). This higher arsenic concentration in surface water was most probably due to the dissolution of realgar and orpiment minerals in Na-borate ore-deposit in the study area. Statistical analytical results of major ion and boron concentration of water were given in Tables $6 \mathrm{a}$ and $\mathrm{c}$ for the wet and dry seasons (Table 6b). Surface water results are in agreement with those from groundwater results and show a strong positive correlation of boron with $\mathrm{Na}$ for the both periods.

Boron concentrations in surface water and groundwater decreased with an increase in the distance from the borate mine area, and concentrations in the dry period were much higher than those in the wet period (Fig. 8).

\subsection{Groundwater-Surface Water Interaction}

Boron concentrations increased to $7 \mathrm{ppm}$ in the Karaören stream (SW3) as a result of the discharge of solid waste of Na-borate which was rapidly dissolved by the rainfall. The boron values in SW6 and SW7 were highly elevated in 2007. The average and maximum boron values in the samples taken from the discharge water of Catören Dam were 6.43 and $9.71 \mathrm{ppm}$, respectively (Table 5). Whereas those values were 1.69 and $2.58 \mathrm{ppm}$ in the sample from discharge of the Kunduzlar Dam (SW12). The average boron value in the water at the meeting point of these two dams' reservoirs (SW13), which forms the Seydisuyu River, was observed as 3.56 ppm (maximum 6.20 ppm). The Seydisuyu River runs
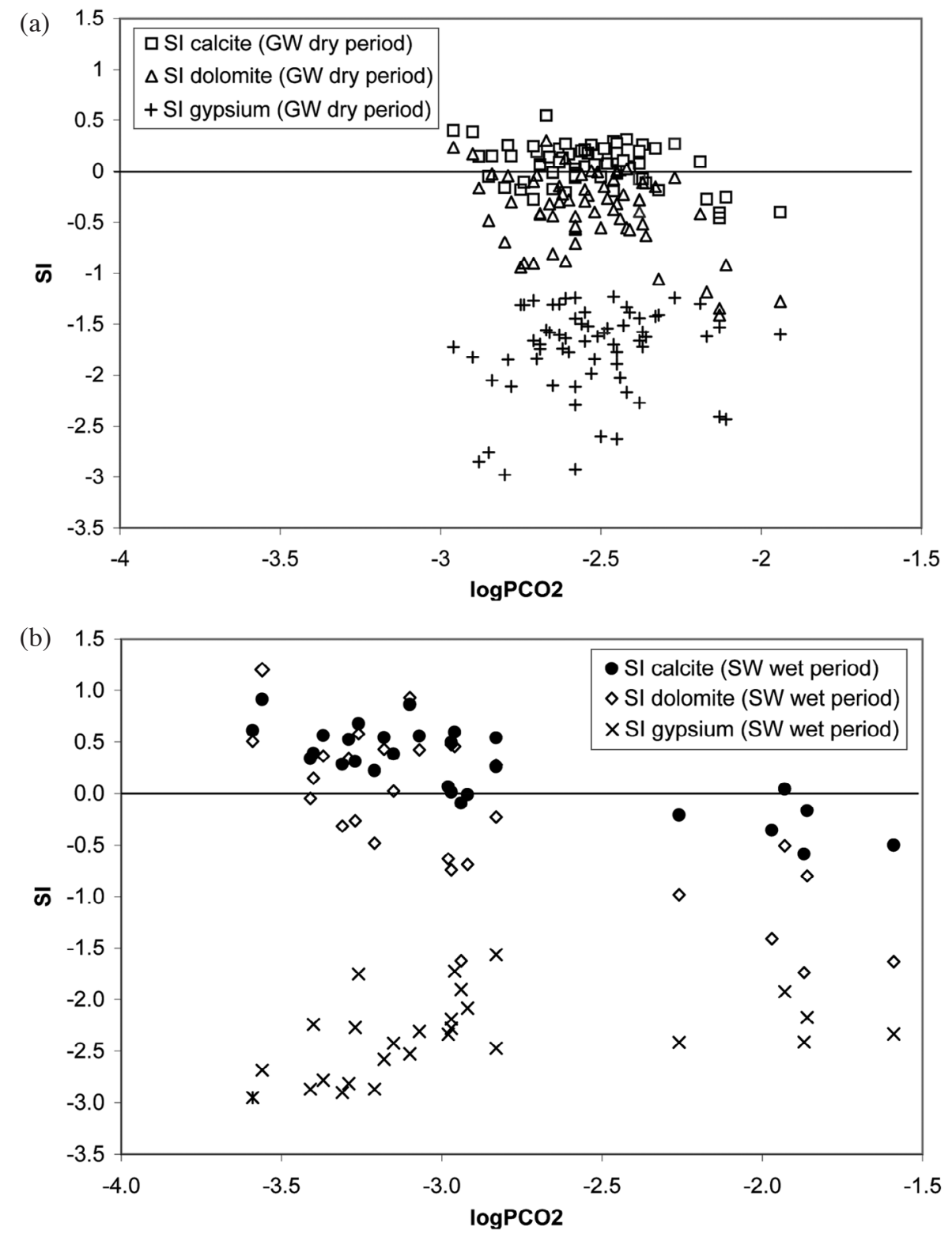

Fig. 6. (a) Chemical equilibrium of groundwater samples on saturation diagram showing relative mineral saturations in wet period. (b) Chemical equilibrium of surfacewater samples on saturation diagram showing relative mineral saturations in wet period. 
Table 4. Analytical results of surfacewater samples.

\begin{tabular}{|c|c|c|c|c|c|c|c|c|c|c|c|c|c|c|c|c|c|}
\hline No & $\begin{array}{l}\text { Sampling } \\
\text { Date }\end{array}$ & pH & $\begin{array}{c}\mathrm{EC} \\
\mathrm{mho} / \mathrm{cm}\end{array}$ & $\mathrm{Na}^{+}$ & $\mathbf{K}^{+}$ & $\mathbf{C a}^{++}$ & $\mathbf{M g}^{++}$ & $\mathrm{CO}_{3}^{--}$ & $\mathrm{HCO}_{3}^{-}$ & $\mathrm{Cl}^{-}$ & $\mathrm{SO}_{4}^{--}$ & B & As & $\mathrm{Sr}$ & $\delta^{18} \mathrm{O}$ & $\delta \mathrm{D}$ & $\mathbf{T}$ \\
\hline SW1 & $2002 \mathrm{~W}$ & 8.20 & 510 & 7.6 & 5.9 & 72.1 & 18.2 & 6.0 & 237.9 & 10.7 & 56.6 & 0.56 & & & & & \\
\hline SW1 & $2003 \mathrm{~W}$ & 8.10 & 320 & 5.6 & 4.2 & 60.3 & 6.8 & 10.8 & 178.1 & 11.7 & 14.9 & 1.20 & & & & & \\
\hline SW1 & 2003 D & 8.23 & 335 & 7.3 & 5.8 & 54.1 & 10.8 & 18.0 & 172.3 & 17.3 & 6.8 & 1.58 & & & & & \\
\hline SW2 & $2002 \mathrm{~W}$ & 8.40 & 520 & 6.4 & 5.9 & 68.1 & 14.6 & 18.0 & 176.9 & 14.2 & 54.2 & 0.64 & & & & & \\
\hline SW2 & $2003 \mathrm{~W}$ & 8.08 & 242 & 5.1 & 2.9 & 41.9 & 6.9 & 3.6 & 145.2 & 11.7 & 6.0 & 1.33 & & & & & \\
\hline SW2 & 2003 D & 8.10 & 390 & 8.8 & 4.0 & 54.9 & 12.1 & 12.8 & 180.7 & 18.2 & 6.8 & 1.85 & & & & & \\
\hline SW3 & $2002 \mathrm{~W}$ & 8.40 & 520 & 8.5 & 7.8 & 73.1 & 15.2 & 18.0 & 262.3 & 14.2 & 8.2 & 0.68 & & & & & \\
\hline SW3 & $2003 \mathrm{~W}$ & 8.00 & 268 & 6.7 & 5.5 & 45.9 & 6.9 & 4.8 & 159.8 & 13.9 & 5.9 & 1.35 & & & & & \\
\hline SW3 & 2003 D & 8.18 & 303 & 14.8 & 7.9 & 43.1 & 9.1 & 18.0 & 152.5 & 18.6 & 5.8 & 2.06 & & & & & \\
\hline SW3 & $2007 \mathrm{~W}$ & 7.27 & 448 & 8.3 & 5.3 & 57.5 & 14.8 & 8.5 & 261.3 & 1.4 & 0.2 & 0.04 & 0.007 & 0.23 & -9.45 & -65.74 & 4.62 \\
\hline SW3 & $2007 \mathrm{D}$ & 8.17 & 350 & 12.7 & 5.1 & 32.8 & 15.9 & 9.3 & 182.4 & 7.5 & 17.6 & 6.85 & 0.020 & 0.76 & -6.7 & -52.91 & 5.78 \\
\hline SW4 & $2002 \mathrm{~W}$ & 8.10 & 520 & 6.0 & 3.5 & 71.1 & 13.4 & 12.0 & 244.0 & 14.2 & 9.6 & 1.09 & & & & & \\
\hline SW4 & $2003 \mathrm{~W}$ & 8.04 & 266 & 6.0 & 2.9 & 42.7 & 10.3 & 4.8 & 161.0 & 11.7 & 8.9 & 2.14 & & & & & \\
\hline SW4 & 2003 D & 7.95 & 365 & 9.5 & 3.4 & 53.1 & 16.4 & 21.0 & 201.3 & 12.4 & 7.2 & 4.36 & & & & & \\
\hline SW5 & $1971 \mathrm{~W}$ & & & & & & & & & & & 18.75 & & & & & \\
\hline SW5 & $1979 \mathrm{~W}$ & & & & & & & & & & & 68.00 & & & & & \\
\hline SW5 & 1979 D & & & & & & & & & & & 58.90 & & & & & \\
\hline SW5 & $1980 \mathrm{~W}$ & & & & & & & & & & & 56.50 & & & & & \\
\hline SW5 & $1980 \mathrm{D}$ & & & & & & & & & & & 57.00 & & & & & \\
\hline SW5 & $1989 \mathrm{~W}$ & & & & & & & & & & & 22.80 & & & & & \\
\hline SW5 & $2002 \mathrm{~W}$ & 8.20 & 480 & 9.4 & 3.1 & 55.1 & 21.3 & 24.0 & 213.5 & 10.7 & 18.7 & 1.38 & & & & & \\
\hline SW5 & $2003 \mathrm{~W}$ & 8.10 & 348 & 5.4 & 3.7 & 61.5 & 10.7 & 7.2 & 206.2 & 18.1 & 7.2 & 2.47 & & & & & \\
\hline SW5 & 2003 D & 8.15 & 378 & 7.1 & 4.1 & 59.6 & 15.5 & 21.0 & 196.7 & 22.2 & 5.5 & 4.19 & & & & & \\
\hline SW6 & $2003 \mathrm{~W}$ & 8.70 & 390 & 37.6 & 4.5 & 44.1 & 9.7 & 72.0 & 91.5 & 15.1 & 20.4 & 24.03 & & & & & \\
\hline SW6 & 2003 D & 8.40 & 593 & 56.8 & 9.0 & 56.9 & 21.9 & 42.0 & 274.5 & 35.1 & 21.7 & 31.11 & & & & & \\
\hline SW6 & $2007 \mathrm{~W}$ & 7.31 & 643 & 36.8 & 5.8 & 60.5 & 27.3 & 0.0 & 390.2 & 10.4 & 25.0 & 36.05 & 0.075 & 2.92 & -9.4 & -65.63 & 1.65 \\
\hline SW7 & $1988 \mathrm{~K}$ & & & & & & & & & & & 1.80 & & & & & \\
\hline SW7 & $1989 \mathrm{~W}$ & & & & & & & & & & & 2.25 & & & & & \\
\hline SW7 & $1999 \mathrm{~W}$ & & & & & & & & & & & 7.80 & & & & & \\
\hline SW7 & $2000 \mathrm{~W}$ & & & & & & & & & & & 3.12 & & & & & \\
\hline SW7 & $2000 \mathrm{D}$ & & & & & & & & & & & 3.75 & & & & & \\
\hline SW7 & $2001 \mathrm{D}$ & 7.65 & 533 & 14.2 & 4.9 & 66.6 & 21.9 & 6.0 & 195.2 & 27.1 & 81.8 & 3.94 & & & & & \\
\hline SW7 & $2002 \mathrm{~W}$ & 8.00 & 583 & 42.1 & 6.0 & 65.8 & 15.4 & 34.0 & 172.8 & 20.2 & 96.0 & 19.73 & & & & & \\
\hline SW7 & 2002 D & 8.54 & 418 & 17.0 & 5.7 & 50.5 & 13.4 & 10.8 & 197.6 & 17.1 & 20.4 & 5.55 & & & & & \\
\hline SW7 & $2003 \mathrm{~W}$ & 8.74 & 410 & 41.2 & 5.3 & 46.5 & 13.6 & 55.2 & 164.7 & 22.7 & 9.0 & 18.25 & & & & & \\
\hline SW7 & 2003 D & 8.30 & 323 & 20.0 & 6.4 & 44.9 & 8.8 & 28.5 & 143.4 & 20.4 & 5.8 & 28.98 & & & & & \\
\hline SW7 & $2007 \mathrm{~W}$ & 6.99 & 574 & 26.9 & 5.0 & 62.7 & 20.4 & 0.0 & 340.6 & 8.4 & 15.8 & 19.48 & 0.056 & 2.38 & -9.25 & -65.97 & 2.64 \\
\hline SW8 & $2001 \mathrm{D}$ & 8.34 & 440 & 15.9 & 6.5 & 43.7 & 20.8 & 15.8 & 122.7 & 22.7 & 75.4 & 4.21 & & & & & \\
\hline SW8 & $2002 \mathrm{~W}$ & 8.43 & 333 & 13.6 & 5.5 & 41.3 & 11.0 & 23.0 & 120.0 & 15.1 & 26.1 & 5.42 & & & & & \\
\hline SW8 & 2002 D & 8.74 & 338 & 16.1 & 5.9 & 38.6 & 9.8 & 15.6 & 114.1 & 20.1 & 30.0 & 5.43 & & & & & \\
\hline SW8 & $2003 \mathrm{~W}$ & 8.48 & 320 & 17.6 & 6.1 & 42.7 & 10.8 & 19.8 & 159.8 & 18.5 & 6.7 & 8.76 & & & & & \\
\hline SW8 & 2003 D & 8.46 & 290 & 16.5 & 5.8 & 39.1 & 7.5 & 28.5 & 123.6 & 16.6 & 4.3 & 9.85 & & & & & \\
\hline SW8 & $2007 \mathrm{~W}$ & 7.52 & 396 & 15.0 & 4.8 & 48.6 & 15.8 & 0.0 & 247.7 & 6.9 & 14.9 & 5.52 & 0.018 & 0.67 & -9.86 & -55.92 & 5.16 \\
\hline SW9 & $1988 \mathrm{D}$ & & & & & & & & & & & 4.80 & & & & & \\
\hline SW9 & $1989 \mathrm{~W}$ & & & & & & & & & & & 3.20 & & & & & \\
\hline SW9 & $2000 \mathrm{~W}$ & & & & & & & & & & & 4.17 & & & & & \\
\hline
\end{tabular}


Table 4. (Continued)

\begin{tabular}{|c|c|c|c|c|c|c|c|c|c|c|c|c|c|c|c|c|c|}
\hline No & $\begin{array}{l}\text { Sampling } \\
\text { Date }\end{array}$ & pH & $\begin{array}{c}\mathrm{EC} \\
\mathrm{mho} / \mathrm{cm}\end{array}$ & $\mathrm{Na}^{+}$ & $\mathbf{K}^{+}$ & $\mathbf{C a}^{++}$ & $\mathbf{M g}^{++}$ & $\mathrm{CO}_{3}^{--}$ & $\mathrm{HCO}_{3}^{-}$ & $\mathrm{Cl}^{-}$ & $\mathrm{SO}_{4}^{--}$ & B & As & $\mathrm{Sr}$ & $\delta^{18} O$ & $\delta \mathrm{D}$ & $\mathbf{T}$ \\
\hline SW9 & $2000 \mathrm{D}$ & & & & & & & & & & & 1.87 & & & & & \\
\hline SW9 & $2001 \mathrm{D}$ & 8.18 & 413 & 10.8 & 5.4 & 43.9 & 21.7 & 10.5 & 157.1 & 16.4 & 57.3 & 3.60 & & & & & \\
\hline SW9 & $2002 \mathrm{~W}$ & 7.97 & 337 & 12.8 & 5.2 & 44.1 & 13.2 & 14.0 & 138.3 & 18.3 & 34.7 & 5.35 & & & & & \\
\hline SW9 & 2002 D & 8.36 & 398 & 14.7 & 5.6 & 41.9 & 15.4 & 10.8 & 154.9 & 26.3 & 24.0 & 5.68 & & & & & \\
\hline SW9 & $2003 \mathrm{~W}$ & 8.28 & 386 & 15.1 & 5.3 & 55.3 & 14.4 & 13.2 & 212.3 & 16.3 & 17.0 & 7.84 & & & & & \\
\hline SW9 & 2003 D & 8.13 & 343 & 18.7 & 5.7 & 43.6 & 12.8 & 16.5 & 176.9 & 21.3 & 6.4 & 9.71 & & & & & \\
\hline SW10 & $1971 \mathrm{~W}$ & & & & & & & & & & & 0.50 & & & & & \\
\hline SW10 & $1979 \mathrm{Y}$ & & & & & & & & & & & 2.50 & & & & & \\
\hline SW10 & $1979 \mathrm{~K}$ & & & & & & & & & & & 2.36 & & & & & \\
\hline SW10 & $1980 \mathrm{Y}$ & & & & & & & & & & & 3.98 & & & & & \\
\hline SW10 & $1980 \mathrm{~K}$ & & & & & & & & & & & 2.50 & & & & & \\
\hline SW10 & $1988 \mathrm{~K}$ & & & & & & & & & & & 2.90 & & & & & \\
\hline SW10 & $1989 \mathrm{Y}$ & & & & & & & & & & & 3.30 & & & & & \\
\hline SW10 & $2001 \mathrm{~K}$ & 8.13 & 515 & 7.5 & 4.5 & 52.9 & 30.4 & 12.0 & 176.9 & 19.6 & 82.9 & 2.27 & & & & & \\
\hline SW10 & $2002 \mathrm{Y}$ & 7.90 & 423 & 6.1 & 3.9 & 53.4 & 16.2 & 4.0 & 170.8 & 17.8 & 44.8 & 1.77 & & & & & \\
\hline SW10 & $2002 \mathrm{~K}$ & 8.30 & 478 & 7.3 & 4.9 & 63.9 & 15.2 & 9.6 & 202.5 & 19.5 & 33.1 & 0.76 & & & & & \\
\hline SW10 & $2002 \mathrm{Y}$ & 7.20 & 480 & 6.2 & 4.7 & 62.1 & 20.7 & 12.0 & 244.0 & 10.7 & 23.5 & 2.72 & & & & & \\
\hline SW10 & $2003 \mathrm{Y}$ & 8.24 & 316 & 5.3 & 4.0 & 50.5 & 11.4 & 14.1 & 158.6 & 16.0 & 13.1 & 2.70 & & & & & \\
\hline SW10 & $2003 \mathrm{~K}$ & 8.00 & 385 & 10.3 & 5.5 & 58.9 & 14.8 & 13.5 & 201.3 & 29.8 & 7.3 & 3.39 & & & & & \\
\hline SW11 & $1986 \mathrm{~W}$ & & & & & & & & & & & 1.95 & & & & & \\
\hline SW11 & $1988 \mathrm{D}$ & & & & & & & & & & & 2.50 & & & & & \\
\hline SW11 & $1989 \mathrm{~W}$ & & & & & & & & & & & 2.65 & & & & & \\
\hline SW11 & $2000 \mathrm{~W}$ & & & & & & & & & & & 1.25 & & & & & \\
\hline SW11 & $2000 \mathrm{D}$ & & & & & & & & & & & 0.41 & & & & & \\
\hline SW11 & $2001 \mathrm{D}$ & 8.51 & 334 & 8.9 & 5.0 & 35.1 & 17.2 & 17.3 & 110.6 & 16.4 & 40.0 & 1.53 & & & & & \\
\hline SW11 & $2002 \mathrm{~W}$ & 8.30 & 297 & 7.3 & 4.0 & 45.3 & 8.0 & 11.0 & 132.2 & 13.0 & 21.0 & 0.92 & & & & & \\
\hline SW11 & $2002 \mathrm{D}$ & 8.70 & 336 & 6.2 & 4.5 & 47.6 & 11.5 & 12.6 & 151.9 & 17.6 & 14.4 & 1.17 & & & & & \\
\hline SW11 & $2003 \mathrm{~W}$ & 8.63 & 267 & 6.8 & 4.4 & 41.3 & 10.0 & 18.0 & 131.2 & 15.3 & 5.2 & 2.21 & & & & & \\
\hline SW11 & $2003 \mathrm{D}$ & 8.28 & 249 & 6.5 & 4.4 & 40.4 & 8.1 & 15.0 & 125.8 & 15.3 & 4.0 & 2.75 & & & & & \\
\hline SW12 & 1999 W & & & & & & & & & & & 1.80 & & & & & \\
\hline SW12 & $2000 \mathrm{~W}$ & & & & & & & & & & & 1.04 & & & & & \\
\hline SW12 & $2000 \mathrm{D}$ & & & & & & & & & & & 0.62 & & & & & \\
\hline SW12 & $2001 \mathrm{D}$ & 8.85 & 298 & 8.7 & 4.9 & 29.3 & 18.6 & 24.0 & 100.7 & 15.5 & 29.0 & 1.39 & & & & & \\
\hline SW12 & $2002 \mathrm{~W}$ & 8.03 & 297 & 5.3 & 3.5 & 43.8 & 10.5 & 10.0 & 142.3 & 11.3 & 18.6 & 1.27 & & & & & \\
\hline SW12 & $2002 \mathrm{D}$ & 8.46 & 324 & 8.4 & 4.8 & 40.9 & 12.3 & 8.4 & 150.1 & 17.0 & 15.2 & 1.19 & & & & & \\
\hline SW12 & $2003 \mathrm{~W}$ & 8.40 & 306 & 7.0 & 4.3 & 46.5 & 12.3 & 14.4 & 164.7 & 16.0 & 5.5 & 2.18 & & & & & \\
\hline SW12 & 2003 D & 8.20 & 258 & 7.3 & 4.4 & 39.1 & 9.3 & 13.5 & 131.2 & 16.0 & 4.4 & 2.58 & & & & & \\
\hline SW12 & $2007 \mathrm{~W}$ & 7.14 & 330 & 8.2 & 4.2 & 44.8 & 14.1 & 0.0 & 216.7 & 3.8 & 10.8 & 1.52 & 0.036 & 0.57 & -7.36 & -61.21 & 4.62 \\
\hline SW13 & $1999 \mathrm{~W}$ & & & & & & & & & & & 8.00 & & & & & \\
\hline SW13 & $2000 \mathrm{~W}$ & & & & & & & & & & & 2.08 & & & & & \\
\hline SW13 & $2000 \mathrm{D}$ & & & & & & & & & & & 2.50 & & & & & \\
\hline SW13 & $2001 \mathrm{D}$ & 8.38 & 378 & 9.6 & 5.4 & 37.4 & 18.1 & 10.5 & 129.6 & 17.3 & 45.2 & 2.25 & & & & & \\
\hline SW13 & $2002 \mathrm{~W}$ & 8.00 & 313 & 6.9 & 3.8 & 45.8 & 9.3 & 10.0 & 134.2 & 13.0 & 26.2 & 1.84 & & & & & \\
\hline SW13 & 2002 D & 8.36 & 368 & 11.6 & 5.3 & 45.1 & 13.3 & 10.8 & 147.6 & 30.6 & 16.3 & 2.55 & & & & & \\
\hline SW13 & $2003 \mathrm{~W}$ & 8.34 & 340 & 10.0 & 4.9 & 50.5 & 13.9 & 16.8 & 184.2 & 16.0 & 9.1 & 4.97 & & & & & \\
\hline SW13 & 2003 D & 8.08 & 295 & 9.4 & 5.0 & 45.1 & 10.6 & 10.5 & 161.7 & 19.1 & 5.9 & 6.20 & & & & & \\
\hline
\end{tabular}


Table 4. (Continued)

\begin{tabular}{|c|c|c|c|c|c|c|c|c|c|c|c|c|c|c|c|c|c|}
\hline No & $\begin{array}{c}\text { Sampling } \\
\text { Date }\end{array}$ & pH & $\begin{array}{c}\mathrm{EC} \\
\mathrm{mho} / \mathrm{cm}\end{array}$ & $\mathrm{Na}^{+}$ & $\mathbf{K}^{+}$ & $\mathbf{C a}^{++}$ & $\mathbf{M g}^{++}$ & $\mathrm{CO}_{3}^{--}$ & $\mathrm{HCO}_{3}^{-}$ & $\mathrm{Cl}^{-}$ & $\mathrm{SO}_{4}^{--}$ & B & As & $\mathrm{Sr}$ & $\delta^{18} O$ & $\delta D$ & $\mathbf{T}$ \\
\hline SW14 & $2001 \mathrm{D}$ & 8.30 & 387 & 11.6 & 6.4 & 37.4 & 18.8 & 16.0 & 176.9 & 17.1 & 8.2 & 3.04 & & & & & \\
\hline SW14 & $2002 \mathrm{~W}$ & 8.20 & 270 & 9.4 & 2.3 & 46.1 & 7.3 & 0.0 & 109.8 & 12.4 & 58.6 & 1.91 & & & & & \\
\hline SW14 & 2002 D & 8.13 & 390 & 13.9 & 5.4 & 44.8 & 11.5 & 10.0 & 144.4 & 25.5 & 24.5 & 2.69 & & & & & \\
\hline SW14 & $2003 \mathrm{~W}$ & 8.30 & 340 & 5.8 & 3.9 & 42.1 & 6.1 & 13.5 & 122.0 & 14.2 & 4.8 & 2.30 & & & & & \\
\hline SW14 & 2003 D & 8.08 & 308 & 13.3 & 5.2 & 43.4 & 10.9 & 12.0 & 161.7 & 22.2 & 4.7 & 5.04 & & & & & \\
\hline SW15 & $2001 \mathrm{D}$ & 8.27 & 370 & 11.1 & 5.6 & 36.8 & 17.0 & 16.0 & 166.7 & 18.4 & 3.7 & 3.04 & & & & & \\
\hline SW15 & $2002 \mathrm{~W}$ & 7.90 & 260 & 9.4 & 2.3 & 51.1 & 2.4 & 0.0 & 122.0 & 12.4 & 41.8 & 1.83 & & & & & \\
\hline SW15 & $2002 \mathrm{D}$ & 8.30 & 343 & 13.9 & 5.2 & 40.8 & 11.5 & 10.0 & 126.1 & 28.4 & 25.1 & 2.23 & & & & & \\
\hline SW15 & $2003 \mathrm{~W}$ & 8.40 & 350 & 6.9 & 4.3 & 40.1 & 9.7 & 15.0 & 115.3 & 24.9 & 5.8 & 3.55 & & & & & \\
\hline SW15 & 2003 D & 8.33 & 278 & 13.4 & 5.2 & 43.9 & 6.4 & 16.5 & 135.7 & 20.0 & 4.4 & 5.62 & & & & & \\
\hline
\end{tabular}

Table 5. The results of the minimum, maximum and average concentrations of boron values in surface water (average values were calculated from different analytical results of the pertinent samples).

\begin{tabular}{|c|c|c|c|c|c|c|c|c|c|}
\hline \multirow{2}{*}{ Sample No } & \multicolumn{4}{|c|}{ Boron (ppm) } & \multirow{2}{*}{ Sample No } & \multicolumn{4}{|c|}{ Boron (ppm) } \\
\hline & Average & Minimum & Maximum & $\mathbf{n}$ & & Average & Minimum & Maximum & $\mathbf{n}$ \\
\hline SW1 & 1.11 & 0.56 & 1.58 & 3 & SW9 & 6.43 & 3.60 & 9.71 & 9 \\
\hline SW2 & 1.27 & 0.64 & 1.85 & 3 & SW10 & 2.27 & 0.76 & 3.39 & 13 \\
\hline SW3 & 2.20 & 0.04 & 6.85 & 5 & SW11 & 1.72 & 0.92 & 2.75 & 10 \\
\hline SW4 & 2.53 & 1.09 & 4.36 & 3 & SW12 & 1.69 & 1.19 & 2.58 & 9 \\
\hline SW5 & 2.68 & 1.38 & 4.19 & 9 & SW13 & 3.56 & 1.84 & 6.20 & 8 \\
\hline SW6 & 30.40 & 24.03 & 36.05 & 3 & SW14 & 3.00 & 1.91 & 5.04 & 5 \\
\hline SW7 & 15.99 & 3.94 & 28.98 & 11 & SW15 & 3.26 & 1.83 & 5.62 & 5 \\
\hline SW8 & 6.53 & 4.21 & 9.85 & 6 & & & & & \\
\hline
\end{tabular}
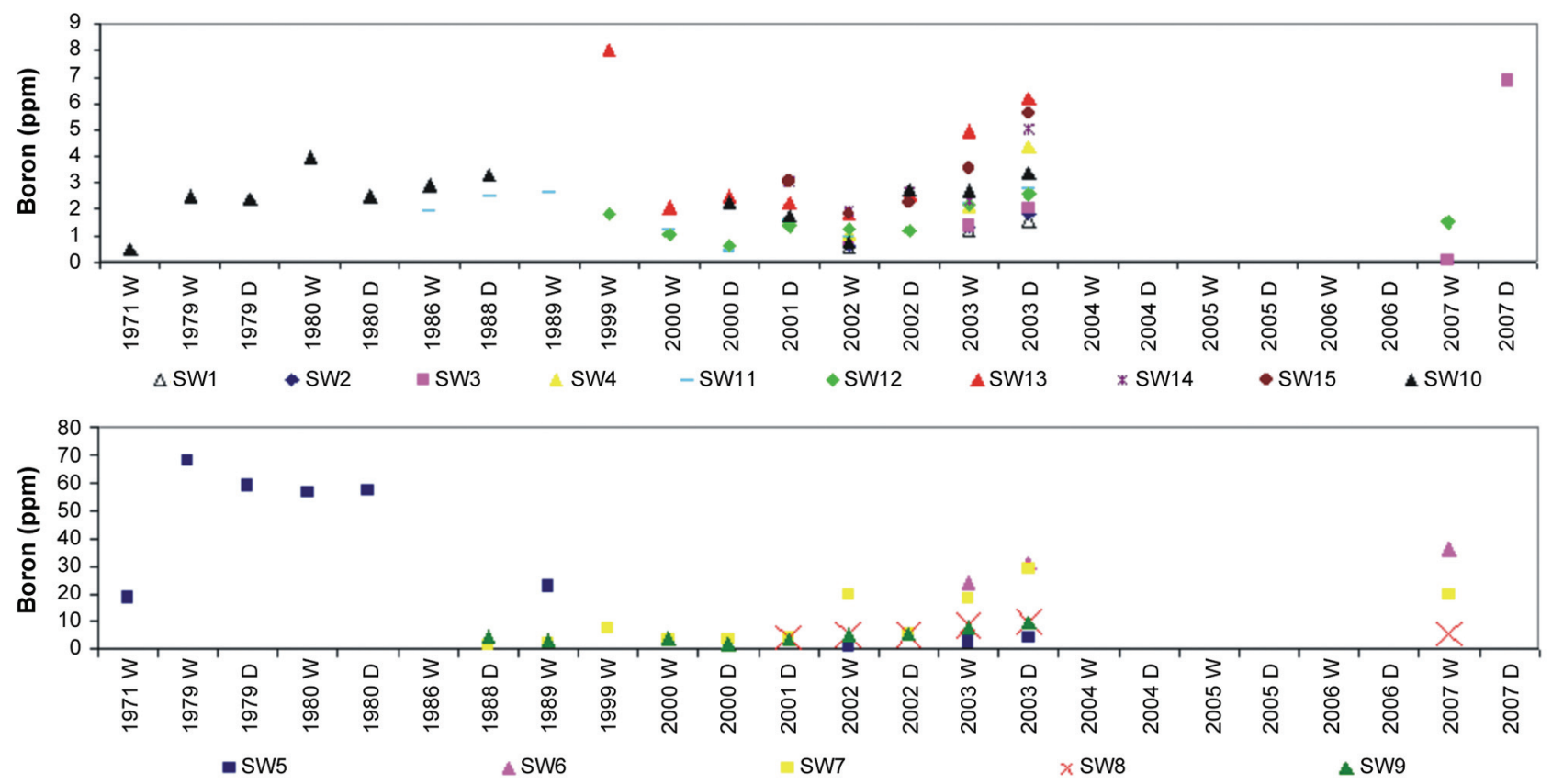

Fig. 7. Boron variations of surface water in time. 
Table 6a. Correlation values of surfacewater (2001 - 2007) (wet period) $(\mathrm{n}=35)$ (values less than 0.5 were omitted).

\begin{tabular}{l|ccccc}
\hline & $\mathbf{p H}$ & $\mathbf{E C}$ & $\mathbf{N a}^{+}$ & $\mathbf{C a}^{++}$ & $\mathbf{M g}^{++}$ \\
\hline $\mathbf{K}^{+}$ & & 0.54 & & & \\
$\mathbf{C a}^{++}$ & & 0.78 & & & \\
$\mathbf{M g}^{++}$ & & 0.82 & & 0.53 & \\
$\mathbf{C O}_{3}^{--}$ & & & 0.57 & & \\
$\mathbf{H C O}_{3}^{-}$ & & 0.74 & & 0.65 & 0.81 \\
$\mathbf{C l}^{-}$ & 0.67 & & & & \\
$\mathbf{S O}_{4}^{--}$ & & & & & \\
$\mathbf{B o r}^{-}$ & & 0.51 & 0.91 & & \\
\hline
\end{tabular}

Table 6b. Correlation values of surfacewater (2001 - 2007) (dry period) $(\mathrm{n}=34)$ (values less than 0.5 were omitted).

\begin{tabular}{l|cccccc}
\hline & $\mathbf{E C}$ & $\mathbf{N a}^{+}$ & $\mathbf{K}^{+}$ & $\mathbf{C a}^{++}$ & $\mathbf{M g}^{++}$ & $\mathbf{C O}_{3}^{--}$ \\
\hline $\mathbf{N a}^{+}$ & & & & & & \\
$\mathbf{K}^{+}$ & & 0.77 & & & & \\
$\mathbf{C a}^{++}$ & 0.61 & & & & & \\
$\mathbf{M g}^{++}$ & 0.77 & & & & & \\
$\mathbf{C O}_{3-}^{--}$ & & 0.67 & 0.52 & & & \\
$\mathbf{H C O}_{3}^{-}$ & 0.67 & & & 0.71 & & \\
$\mathbf{C l}^{-}$ & & 0.53 & & & & \\
$\mathbf{S O}_{4}^{--}$ & 0.63 & & & & 0.75 & \\
$\mathbf{B o r}$ & & 0.82 & 0.59 & & & 0.74 \\
\hline
\end{tabular}

Table $6 c$. Correlation values of surfacewater (2007) (wet period) $(n=5)$ (values less than 0.5 were omitted).

\begin{tabular}{l|cccccccccc}
\hline & $\mathbf{p H}$ & $\mathbf{E C}$ & $\mathbf{N a}^{+}$ & $\mathbf{K}^{+}$ & $\mathbf{C a}^{++}$ & $\mathbf{M g}^{++}$ & $\mathbf{C O}_{3}^{--}$ & $\mathbf{C l}^{-}$ & $\mathbf{S O}_{4}^{-{ }^{-}}$ & $\mathbf{B o r}$ \\
\hline $\mathbf{N a}^{+}$ & 0.69 & & & & & & & & & \\
$\mathbf{C a}^{++}$ & & 0.86 & & 0.79 & & & & & & \\
$\mathbf{M g}^{++}$ & & 0.63 & & 0.56 & 0.80 & & & & & \\
$\mathbf{C O}_{3}^{--}$ & 0.96 & & 0.77 & & & & & & \\
$\mathbf{H C O}_{3}^{-}$ & & 0.59 & & 0.72 & 0.85 & 0.97 & & & \\
$\mathbf{C l}^{-}$ & 0.76 & & 0.95 & & & & 0.77 & & \\
$\mathbf{S O}_{4}^{--}$ & & & 0.78 & & & & & 0.90 & \\
$\mathbf{B o r}$ & 0.58 & & 0.97 & & & & 0.67 & 0.86 & 0.70 & \\
$\mathbf{A s}$ & & & & & & & 0.63 & & & \\
$\mathbf{S r}$ & 0.57 & & 0.98 & & & & 0.68 & 0.87 & 0.70 & 0.99 \\
\hline
\end{tabular}

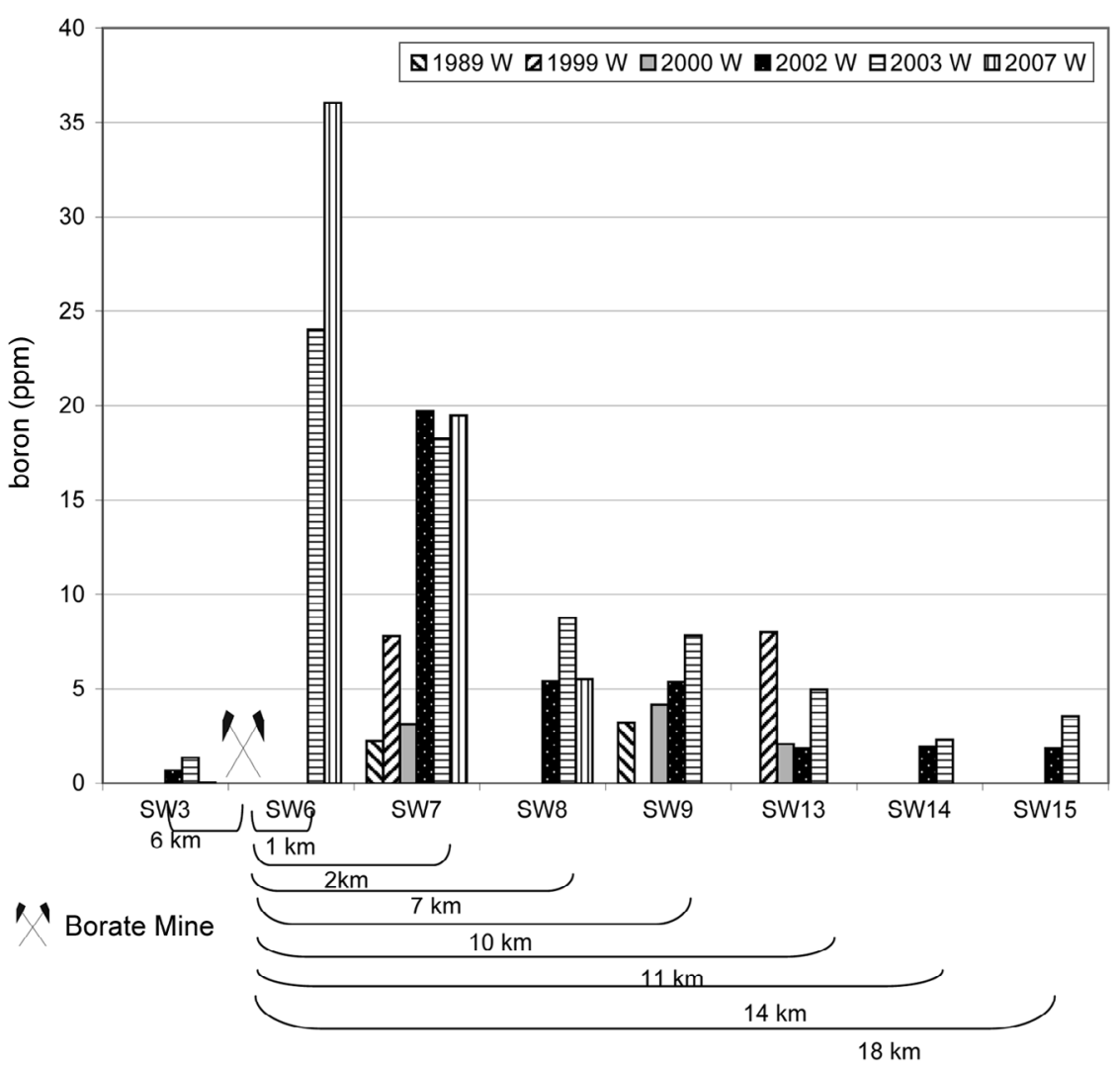

Fig. 8. Boron variations in distance from the Na-borate mine. 
to the Kesenler regulator (Fig. 1) where the water is diverted into two irrigation canals as the right and left side (SW14 and SW15, respectively) on the Seyitgazi Plain. The average and maximum boron concentrations of surface water in the approach to the Seyitgazi Plain were 3 and $5 \mathrm{ppm}$ in SW14 and 3.26 and 5.62 ppm in SW15, respectively. Thus, the boron concentration in the surface water at the discharge area of the Na-borate mine has been affected.

Both ground and surface water in the study area are generally of a Ca- $\mathrm{HCO}_{3}$ type (Figs. 9a and b). Significant positive correlation was found between boron and arsenic and strontium in both for surface water and groundwater (Figs. 10a and b). Because arsenic and strontium salts are generally found in the same playa lake (i.e., Kirka borax province) as associated with boron. The positive correlation shows that increasing concentrations of one constituent are associated with increasing concentrations of another constituent and they may migrate under similar conditions (Senior and Sloto 2006). Boron concentration in water samples taken from the catchment area, SW7, SW8, SW9, SW12, SW13, SW14 and SW15, increased in the different rates (between 2 and 7 times) from the dry period of 2001 to the dry period of 2003. Parallel to the boron increase in surface water, boron also elevated in the dry period between 2001 and 2003. This may be indicative of the groundwater-surface water interaction in the study area. On the other hand, arsenic concentrations in surface water were in higher concentrations up to $0.075 \mathrm{ppm}$ that is 7.5 times higher than the allowable limit for drinking water, given by WHO, EPA and TS 266.

\subsubsection{Stable Isotopes and Tritium}

Isotope samples were collected from the study area to determine relationships between groundwater and surface water as well as to identify that of recharge and discharge. The $\delta^{18} \mathrm{O}$ values of the groundwater have varied from -8.66 to $-9.86 \%$ for the wet season and from -9.02 to $-9.98 \%$ o for the dry season. However, there is a slight deviation in stable isotope values of surface water (in SW3) in the dry season due to the enrichment by the evaporation. The evaporation effect has also been seen for SW8 and SW12 even in the wet season (Fig. 11). In contrast to general expectations, groundwater at the Seyitgazi Plain has slightly more negative stable isotope values (GW22, GW18, GW14) in the dry period in comparision with those of the wet period. Given the seasonal variation in stable isotope values of groundwater, relatively positive values in the wet period may be due to the influence of surface water which infiltrated into the shallow aquifer system from the previous irrigation season (in the dry period) and that water from the lake of the Catören and Kunduzlar dams has more positive stable isotope values (SW8 and SW12) because of an evaporation effect (Fig. 11). However, above the Catören dam, surface water at the section of SW6 and SW7 are close to similar stable isotope values of groundwater at upstream (GW41) and downstream (GW2, GW14, GW18, GW22) areas (Tables 1 and 4). Tritium values of surface water are generally higher (between 4.62 and 5.78 T.U.) than that of groundwater which refer to a long residence time due to deep circulation at depth. Tritium values of surface water taken from SW6 and SW7, somehow, are of lower than aforementioned values for surface water. The lower tritium values at SW6 and SW7 (1.65 and 2.64 T.U.) may be due to the mixing of groundwater which has much lower tritium values. Possible interaction between groundwater and surface water at the upstream area of the dams are another alternative to explain high boron and arsenic concentrations in the dams' water besides directly leaching from boron disposal from the mining activites. The interaction of groundwater and surface water at SW7 and SW8 can also be seen in tritium $-\delta^{18} \mathrm{O}$ and $\delta \mathrm{D}$ in Figs. 12a and b, respectively. One can easily recognize that all groundwater samples are almost clustered in the same area (icluding SW6 and SW7 at the some extent) which are distinctly plotted far from the surface water samples (SW3, SW8, SW8). In fact, stable isotope composition of groundwater at the Seyitgazi Plain during the dry season was expected to be notably enriched due to infiltration of irrigation water into the aquifer system. However, the enrichment in stable isotope values of groundwater at the Seyitgazi Plain was not strongly observed. Because, it is most possibly, irrigation water from the Catören dam was not available due to the extreme drought period in 2007. On the other hand, the stable isotope results of GW41 (located upstream of the Seyitgazi Plain), GW2, GW18 and GW22 (at the Seyitgazi Plain) have a similar stable isotope and tritium abundances. Therefore, this may imply that groundwater in the Seyitgazi Plain has been replenished from Kirka Province which is consistent with the conceptual model given in Fig. $2 b$.

\section{CONCLUSIONS}

The surface water and groundwater resources are contaminated by Na-borate mine activities in addition to the natural leaching from the boron-bearing formation at the catchment area of the study area. The analytical results of surface water and groundwater samples taken from the recharge and discharge areas demonstrate that boron concentrations have been increasing since 1989. The increasing boron concentration in the surface water is widely associated with the Naborate mine, while the increase in groundwater is as a result of the utilization of surface water in a high boron concentration for irrigation purposes on the Seyitgazi Plain. The recent boron concentration in groundwater at Seyitgazi Plain is three-four times higher than initial boron contents $(0.1$ $0.5 \mathrm{ppm})$. This higher boron content in the groundwater of the Seyitgazi Plain is mostly due to replenishment from surface water by the infiltration of irrigation water supplied 
(a)

100

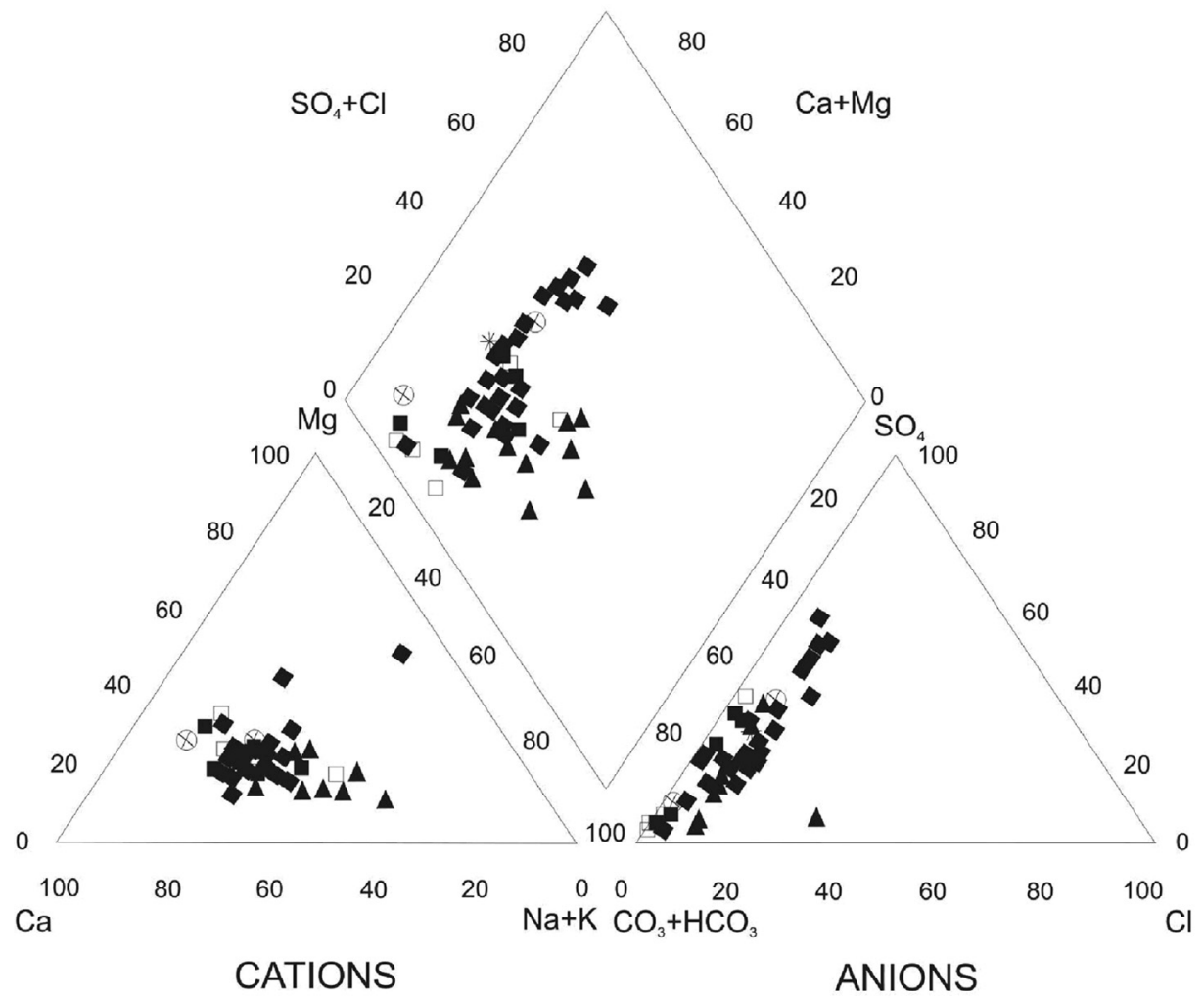

(b)

100

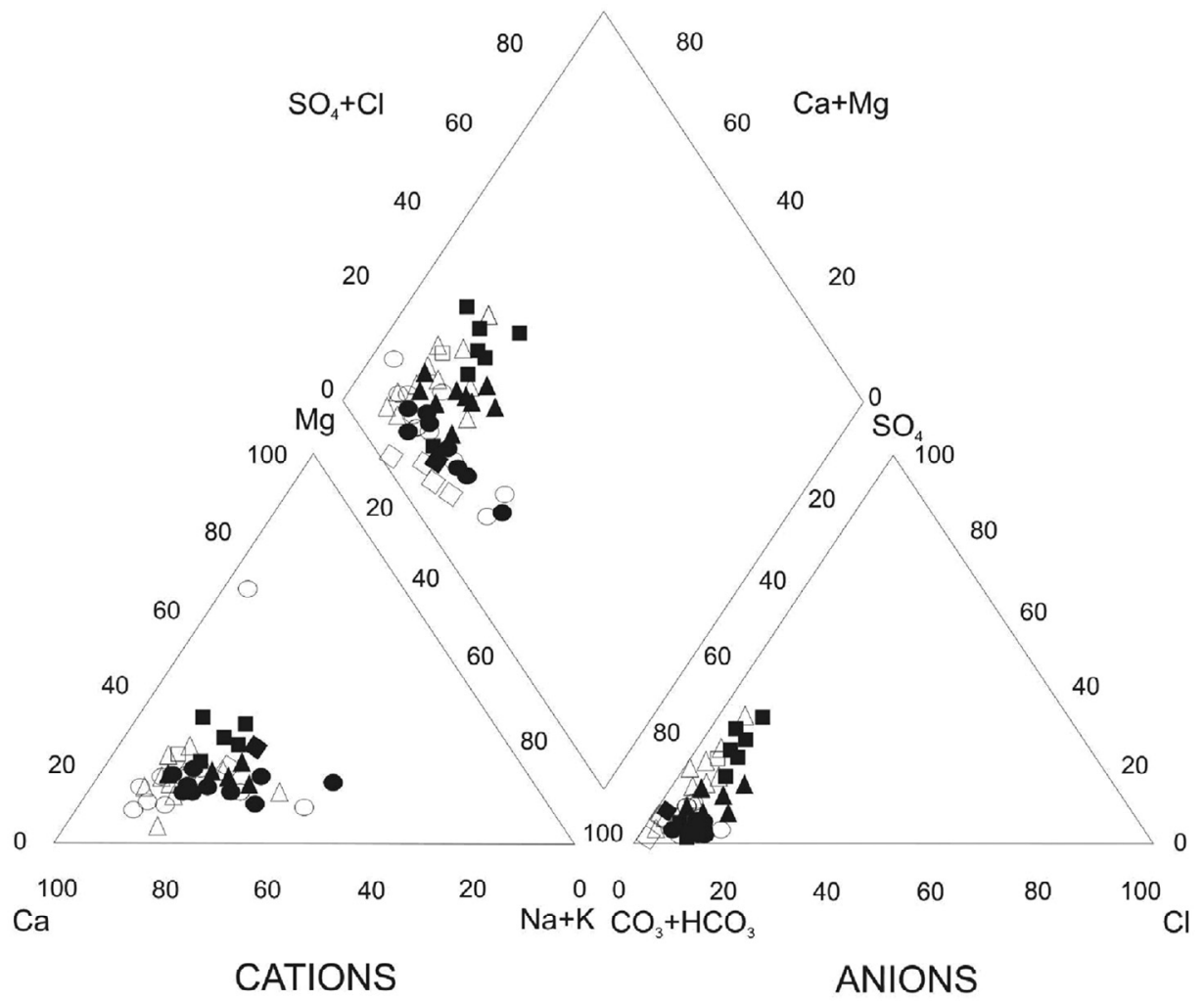

Fig. 9. (a) Piper diagram for groundwater. (b) Piper diagram for surfacewater. 

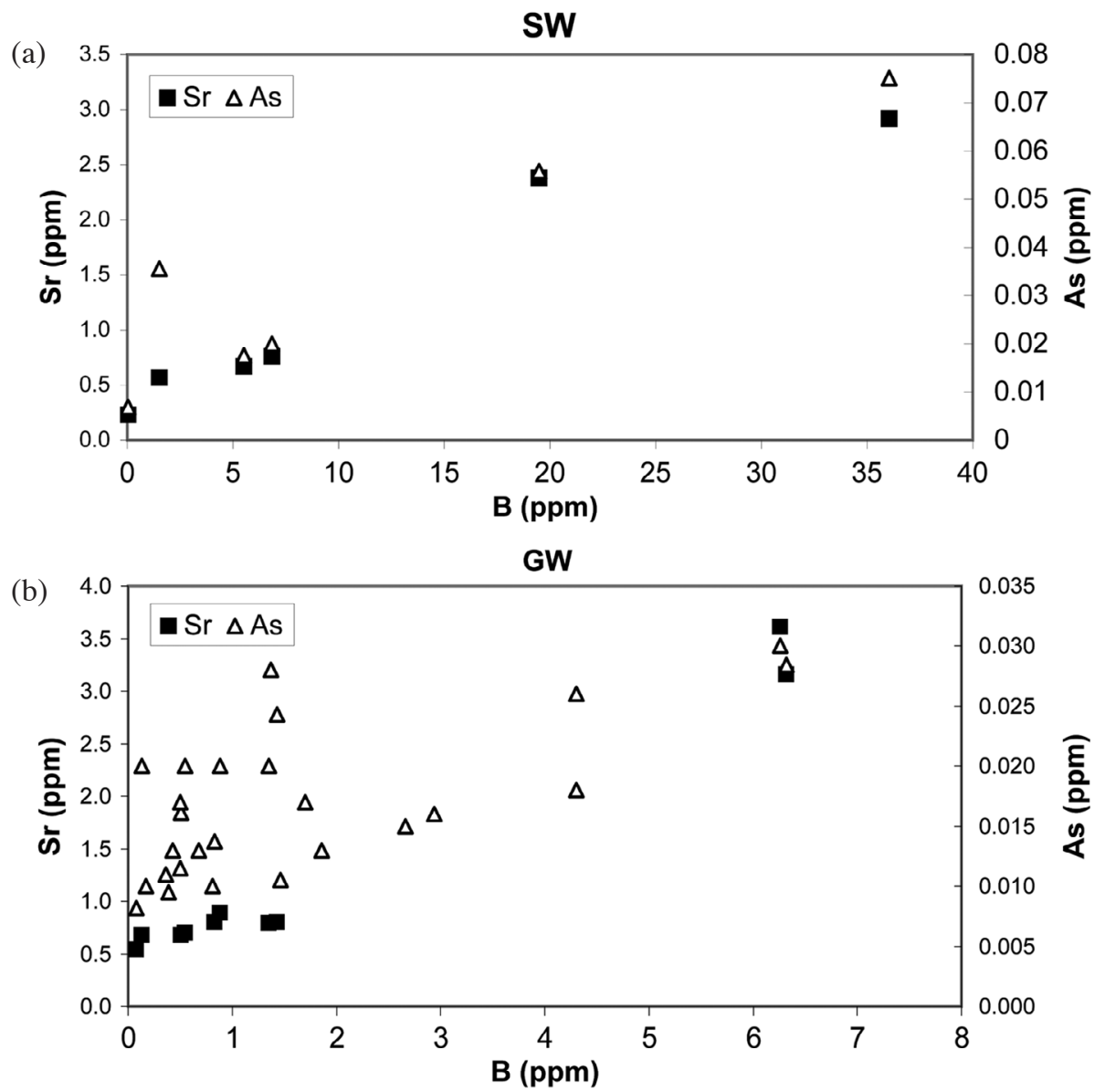

Fig. 10. (a) B-Sr-As graph for surface water. (b) B-Sr-As graph for groundwater.

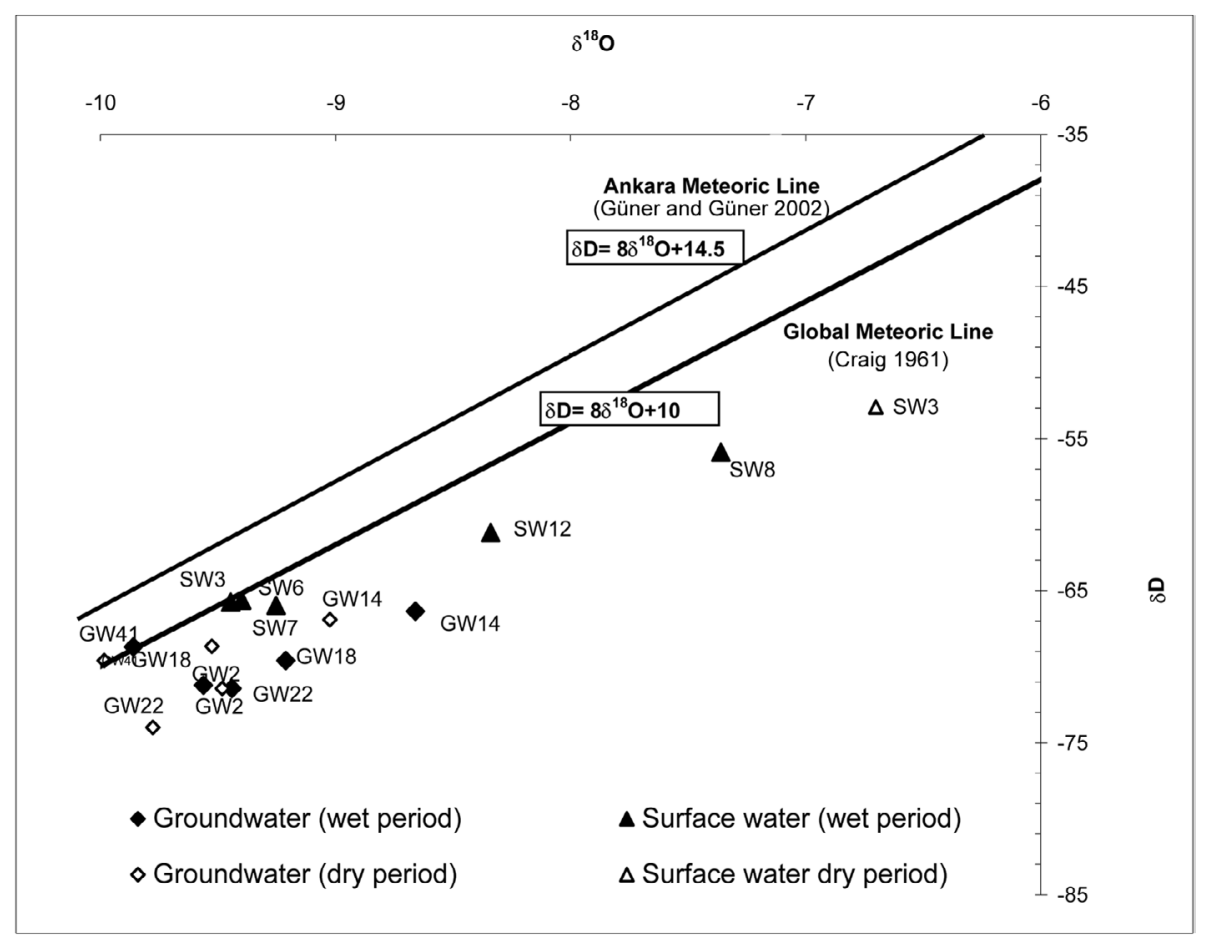

Fig. $11 . \delta^{18} \mathrm{O}-\delta \mathrm{D}$ graph. 

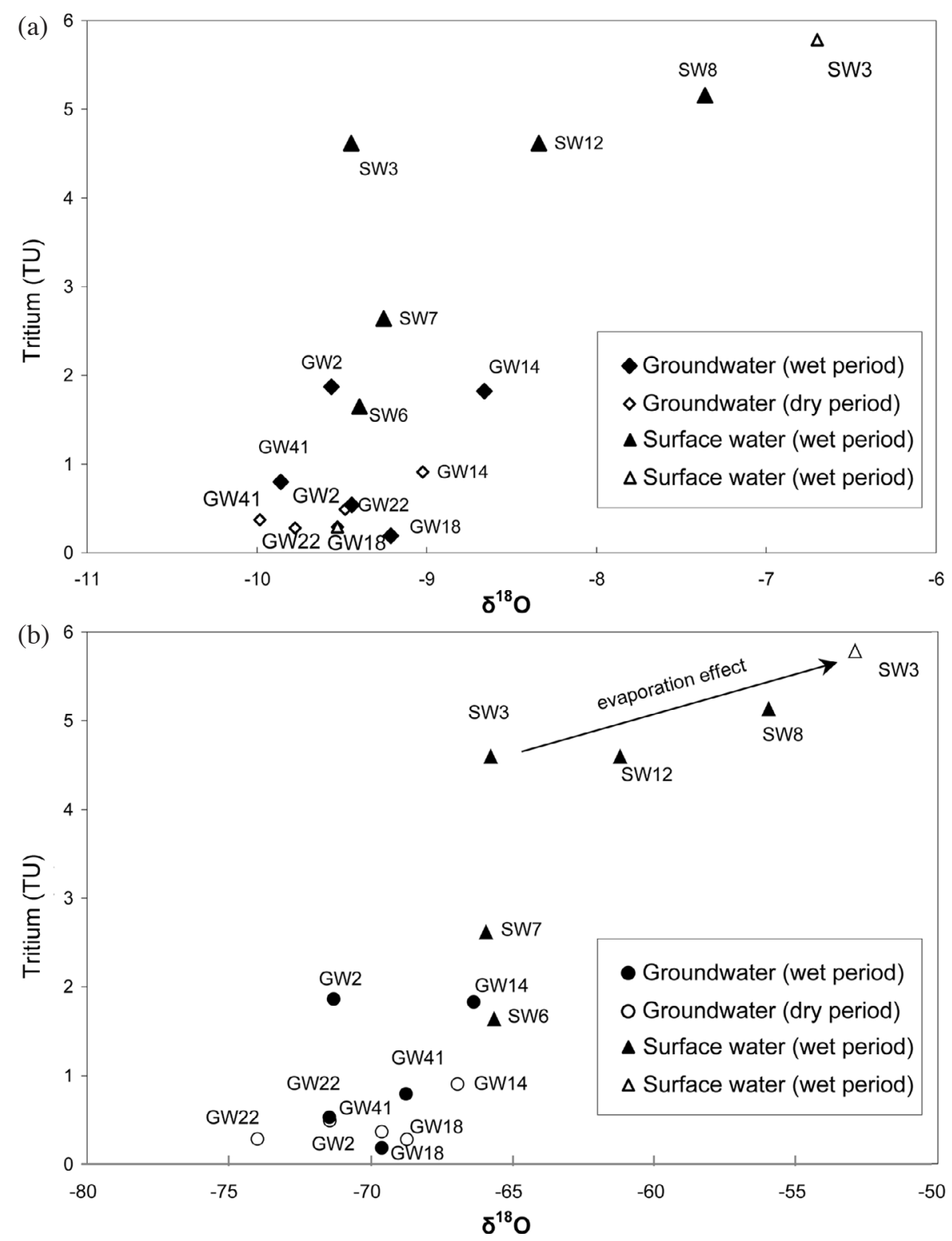

Fig. 12. (a) $\delta^{18} \mathrm{O}-{ }^{3} \mathrm{H}$ graph. (b) $\delta \mathrm{D}-{ }^{3} \mathrm{H}$ graph.

from the Catören dam located in the catchment area of the Seyitgazi Plain. Concordantly, between 2003 and 2007, boron concentration remarkably increased more than one ppm in groundwater in the Seyitgazi Plain. The boron concentration of the Seydisuyu River was also higher than the recommended limit for irrigation purposes which should be less than one ppm according to Ayers and Westcot (1976). High boron content in water resources in the study area are mostly related to the Na-borate mine activities at the Kirka Province. The arsenic concentration in the groundwater of the Seyitgazi Plain was also more than the allowable limit for drinking purpose which is recommended as $10 \mu \mathrm{g} \mathrm{l}^{-1}$ by EPA (2001). During the dry period, boron, arsenic and strontium contents in water were relatively higher than the wet period.
Groundwater in the study area is generally in $\mathrm{Ca}-\mathrm{HCO}_{3}$ type. The high $\mathrm{pH}$ value of water likely contributes to the mobilization of boron, locally. Irrigation from Catören dam's reservoir strongly affects the local geohydrochemical conditions and might be an important factor for boron mobilization in the study area.

Chemical analysis shows that the groundwater of the Seyitgazi Plain is influenced by the Na-borate mine activities in the catchment area. High boron concentration of surface water in the catchment area negatively affects the quality of groundwater of the Seyitgazi Plain by means of surface water-groundwater interaction since water with high boron content comes from the Catören dam used for irrigation purposes on the Seyitgazi Plain. Water-rock interaction within the Sarıkaya formation, which contains higher borate 
minerals, causes increasing boron concentrations in the groundwater in Kirka Province. Conformingly, the boron concentration reached up to $7.82 \mathrm{ppm}$ in the deep well (GW41: $172 \mathrm{~m}$ ) located near the Kirka boron mine area. After leaching Na-borate from the Kirka borate deposit, the disposal mine waste has been deposited in tailing areas next to the mine site. Since the mine waste has a higher boron content, it is readily dissolved in aquatic environment under the atmospheric condition (i.e., rain and snow). Eventually, this high boron-contained water is mixed with surface water (Özkurt 2000). Thus, boron concentration increases in the wet season in streams and creeks close to the tailing site. It is obvious that these streams and creeks recharge into the Catören dam's reservoir which is used for irrigation water on the Seyitgazi Plain. Because groundwater flow direction is from Kirka to Seyitgazi, it also contributes to the increase of boron content of the groundwater in the Seyitgazi Plain.

In conclusion, water coming from the Catören dam is recharged from the surface water in contact with the tailings of Kirka Na-borate mine. The groundwater flow direction from the Kirka to Seyitgazi Plain possibly contributes to the increase of boron content of the groundwater in the Seyitgazi Plain. Arsenic content in groundwater exceeds the WHO and EU limits for the safe drinking water standard $\left(10 \mu \mathrm{g}^{-1}\right)$, especially for water from GW22 and GW26 at the discharge area of Kirka Province due to either naturally leached from tuff or boron-bearing formation. Isotope analytical results also confirm that groundwater at the Seyitgazi Plain are hydraulically linked with the ground water in Kirka Province. Therefore, any environmental contamination in the Kirka Province will affect the groundwater quality of the Seyitgazi Plain in addition to surface water influence. Periodically, water samples should be collected for monitoring boron and arsenic levels in the water resources as well as required precautions taken to prevent further water quality deterioration in the study area.

Acknowledgements The authors would like to thank the staff in the Regional Directorate of State Hydraulic Works (DSI) and Provincial Bank (Iller Bankası) in Eskisehir. We are also indebted to Erdogan Ayday for giving permission to use the previous data related to the study area. The authors are indebted to Dr. Francesco Italiano and another anonymous reviewer whose insightful comments and suggestions substantially improved this paper.

\section{REFERENCES}

Akbas, F., 1998: The impact of the different boron concentration on the productivity of cotton and its accumulation in soil, General Directorate of Rural Service, Local publications of Menemen Research Institute. (in Turkish)

Ayers, R. S. and D. W. Westcot, 1976: Water Quality for Agriculture. FAO Irrigation and Drainage Paper, ver- sion 29, available at: http://www.fao.org/DOCREP/ 003/T0234E/T0234E00.HTM.

Baba, A., G. Yuce, O. Deniz, and D. Y. Ugurluoglu, 2009: Hydrochemical and isotopic composition of Tuzla geothermal field (Canakkale-Turkey) and its environmental impacts. Environ. Forensics, 10, 144-161, doi: 10.1080/15275920902873418. [Link]

Baysal, O., 1972: Tunelite, a new hydrous strontium borate from the Sarikaya borate deposits in Turkey. M. T. A. J., 79, 22-29.

Culver, B. D., R. G. Smith, R. J. Brotherton, P. L. Strong, and T. M. Gray, 1994: Chapter 44: Boron. In: Clayton, G. D. and F. E. Clayton (Eds.), Patty's Industrial Hygiene and Toxicology, New York, John Wiley and Sons, volume 2, part 1.

Demirtas, A., 2006: Boron compounds and their potential of use in agriculture. Ataturk Univ. Agr. Fac. J., 37, $111-115$.

Deutsch, W. J., 1997: Groundwater Geochemistry: Fundamentals and Applications to Contamination. Lewis Publisher, USA, 232 pp.

DSI (the Regional Directorate of State Hydraulic Works), 1971: Hydrogeologic research report of Kirka and its vicinity. General Directorate of State Hydraulic Works, Ankara. (in Turkish)

DSI (the Regional Directorate of State Hydraulic Works), 1983: General report of boron contamination in the Kirka region. General Directorate of State Hydraulic Works, Ankara. (in Turkish)

ECETOC (European Centre for Ecotoxicology and Toxicology Of Chemicals), 1997: Ecotoxicology of some inorganic borates - Interim report, Brussels, special report No. 11.

EPA (United States Environmental Protection Agency), 2001: Environmental Protection Agency Publication, 816-R-01-003.

Floyd, P. A., C. Helvaci, and S. K, Mittwede, 1998: Geochemical discrimination of volcanic rocks associated with borate deposits: An exploration tool? J. Geochem. Explor., 60, 185-205, doi: 10.1016/S0375-6742(97)00 047-2. [Link]

Gemici, Ü. and G. Tarcan, 2002: Distribution of boron in thermal waters of western Anatolia, Turkey, and examples of their environmental impacts. Environ. Geol., 43, 87-98, doi: 10.1007/s00254-002-0608-x. [Link]

Gemici, Ü., G. Tarcan, C, Helvaci, and M. Somay, 2008: High arsenic and boron concentrations in groundwaters related to mining activity in Bigadiç borate deposits (Western Turkey). Appl. Geochem., 23, 2462-2476, doi: 10.1016/j.apgeochem.2008.02.013. [Link]

Güner, F. G. and I. N. Güner, 2002: Determination of hydrogeology of the karstic springs of Sakarbasi by using hydrochemistry and environmental isotope techniques. $1^{\text {st }}$ Symposium on the utilization of isotope techniques 
in hydrology, 21-25 October 2002, Adana, 207-228.

Helvaci, C. and R. N. Alonso, 2000: Borate deposits of Turkey and Argentina: A summary and geological comparison. Turkish J. Earth Sci., 9, 1-27.

Helvaci, C., H. Mordogan, M. Çolak, and I. Gündogan, 2004: Presence and distribution of lithium in borate deposits and some recent lake waters of west-central Turkey. Int. Geol. Rev., 46, 177-190. doi: 10.2747/00206814.46.2.177. [Link]

Hem, J. D., 1975: Study and Interpretation of the Chemical Characteristics of Natural Water. US Geological Survey, Second Ed., Water-Supply Paper, 1473, 361.

Iller Bankas1, 2000: Drinking water facility of Doğancayır (Eskisehir) Municipality (Eskisehir). Regional Directorate of Iller Bankası, Eskisehir. (in Turkish)

Mellor, J. W., 1980: A Comprehensive Treatise on Inorganic and Theoretical Chemistry. Volume 5, London, New York, Longman, Green.

Onacak, T., 1990: The impact of the water having high boron content on surface water of Eskisehir region. Master Thesis, Hacettepe University, Ankara. (in Turkish)

Ozcan, A., M. C. Göncüoğlu, and N. Turan, 1989: Geology of Kütahya-Çifteler-Bayat-İhsaniye Area. General Directorate of Mineral Research and Exploration (MTA) Journal, Ankara.

Özkurt, S., 2000: Boron accumulation in carp tissues (Cyprinus carpio L., 1758) in Dam Lakes Çötören and Kunduzlar (Kirka-Eskişehir). Turkish J Biol., 24, 663676. (in Turkish)

Palmer, M. R. and C. Helvaci, 1995: The boron isotope geochemistry of the Kirka borate deposit, Western Turkey. Geochim. Cosmochim. Acta, 59, 3599-3605, doi: 10.1016/0016-7037(95)00227-Q. [Link]

Palmer, M. R. and C. Helvaci, 1997: The boron isotope geochemistry of the Neogene borate deposits of Western Turkey. Geochim. Cosmochim. Acta, 61, 3161-3169, doi: 10.1016/S0016-7037(97)00135-X. [Link]

Rural Service of Eskisehir Research Institute (Köy Hizmetleri Eskisehir Araştirma Enstitüsü), 2001: Examination of boron content in Eskisehir-Seyitgazi Irrigation Network and mapping with GIS, Project No: 01240E01, 2001 Final Report.

Rural Service of Eskisehir Research Institute (Köy Hizmetleri Eskisehir Araştirma Enstitüsü), 2002: Examination of boron content in Eskisehir-Seyitgazi Irrigation Network and mapping with GIS, Project No: 01240E01, 2002 Final Report.

Rural Service of Eskisehir Research Institute (Köy Hizmetleri Eskisehir Araştirma Enstitüsü), 2003: Examination of boron content in Eskisehir-Seyitgazi Irrigation Network and mapping with GIS. Project No: 01240E01, 2003 Final Report.

Senior, L. A. and R. A. Sloto, 2006: Arsenic, boron, and fluoride in ground water in and near diabase intrusions,
NewarkBasin, southeastern Pennsylvania. US Geological Survey Scientific Investigations Report 2006-5261.

Smedley, P. L. and D. G. Kinniburgh, 2002: A review of the source, behaviour and distribution of arsenic in natural waters. Appl. Geochem., 17, 517-568, doi: 10.1016/S0 883-2927(02)00018-5. [Link]

Ugurluoglu, D., 2004: The effects of borate deposit in Kirka-Eskisehir region on the boron content of the surface and groundwater in the region and its variations with time. Master Thesis, Institute of Science and Technology, Osmangazi University, Eskisehir. (in Turkish)

Ugurluoglu, D. and G. Yuce, 2004: The temporal variation of boron content in surface and groundwater sourced from borate deposits. In: The Kirka (Eskisehir) Region, $58^{\text {th }}$ Geological Congress of Turkey Extended Abstracts Book, 227-228. (in Turkish)

Unsal, A. and S. Metintas, 2002: Evahıation of admissions to health units because of exposure to boron in Kırka (Eskişehir) preliminary report CEVKOR, 11, 44, 7-10. (in Turkish)

Urgüplü, M., 1985: Borate deposits in Balıkesir region and its contamination effects on the soils and preventation of contamination, Directorate of State Hydraulic Works XVIII, 9-13 September 1985, Edirne. (in Turkish)

Vengosh, A., C. Helvaci, and I. H. Karamanderesi, 2002: Geochemical constraints for the origin of thermal waters from western Turkey. Appl. Geochem., 17, 163183, doi: 10.1016/S0883-2927(01)00062-2. [Link]

WHO (World Health Organization), 1998: Boron: Environmental health criteria, 204, ISSN 0250-863X.

Wolkersdorfer, C., 1996: Hydrogeochemical investigations of an abandoned uranium mine in the Erzgebirge/Germany. Appl. Geochem., 11, 237-241, doi: 10.1016/08 83-2927(95)00060-7. [Link]

Yalcin, H. and O. Baysal, 1991: Geologic possition, distribution and occurance of borate deposits in Kırka (Seyitgazi). Gen. Direct. Mineral Res. Explor. (MTA) J., 113, 93-104. (in Turkish)

Yasin, U. D. and G. Yuce, 2008: The effect of drought on the quality of water resources mixed with leachate waters from an open-pit boraxe mine. $10^{\text {th }}$ International Mine Water Association Congress, Mine Water and the Environment June 2-5, 2008, Karlovy Vary, Czech Republic.

Younger, P. L., C. Wolkersdorfer, and ERMITE-Consortium, 2004: Mining impacts on the fresh water environment: Technical and managerial guidelines for catchment scale management. Mine Water Environ., 23, s2-s80, doi: 10.1007/s10230-004-0028-0. [Link]

Zheng, M. and X. Liu, 2009: Hydrochemistry of Salt Lakes of the Qinghai-Tibet Plateau, China. Aquat. Geochem., 15, 293-320, doi: 1001-7410.0.1990-01-002, doi: 10. 1007/s10498-008-9055-y. [Link] 